CRYSTALLOGRAPHIC COMMUNICATIONS

ISSN 2056-9890

Received 31 May 2019

Accepted 14 June 2019

Edited by W. T. A. Harrison, University of Aberdeen, Scotland

Keywords: crystal structure; polyoxomolybdates; IR spectroscopic study; hydrogen bonding.

CCDC reference: 1922927

Supporting information: this article has supporting information at journals.iucr.org/e

\section{Synthesis and crystal structure of a new hybrid organic-inorganic material containing neutral molecules, cations and heptamolybdate anions}

\author{
Bougar Sarr, ${ }^{\text {a* }}$ Abdou Mbaye, ${ }^{\mathrm{b}}$ Wally Diallo, ${ }^{\text {a }}$ Cheikh Abdoul Khadir Diop, ${ }^{\text {a }}$ \\ Mamadou Sidibe ${ }^{a}$ and Francois Michaud ${ }^{c}$
}

'Laboratoire de Chimie Minérale et Analytique, Département de Chimie, Faculté des Sciences et Téchniques, Université
Cheikh Anta Diop, Dakar, Senegal, 'baboratoire de Chimie et de Physique des Matériaux (LCPM) de I’Université Assane
Seck de Ziguinchor (UASZ), BP 523 Ziguinchor, Senegal, and '⿳㇒Service Commun d'Analyse par Diffraction des Rayons X,
Universite de Bretagne Occidentale, 6, avenue Victor Le Gorgeu, CS 93837, F-29238 Brest cedex 3, France.
*Correspondence e-mail: bouks89@gmail.com

The title compound, hexakis(2-methyl- $1 H$-imidazol-3-ium) heptamolybdate 2-methyl- $1 \mathrm{H}$-imidazole disolvate dihydrate, $\left(\mathrm{C}_{4} \mathrm{H}_{7} \mathrm{~N}_{2}\right)_{6}\left[\mathrm{Mo}_{7} \mathrm{O}_{24}\right] \cdot 2 \mathrm{C}_{4} \mathrm{H}_{6} \mathrm{~N}_{2} \cdot-$ $2 \mathrm{H}_{2} \mathrm{O}$, was prepared from 2-methylimidazole and ammonium heptamolybdate tetrahydrate in acid solution. The $\left[\mathrm{Mo}_{7} \mathrm{O}_{24}\right]^{6-}$ heptamolybdate cluster anion is accompanied by six protonated $\left(\mathrm{C}_{4} \mathrm{H}_{7} \mathrm{~N}_{2}\right)^{+}$2-methylimidazolium cations, two neutral $\mathrm{C}_{4} \mathrm{H}_{6} \mathrm{~N}_{2}$ 2-methylimidazole molecules and two water molecules of crystallization. The cluster consists of seven distorted $\mathrm{MoO}_{6}$ octahedra sharing edges or vertices. In the crystal, the components are linked by $\mathrm{N}-\mathrm{H} \cdots \mathrm{N}, \mathrm{N}-$ $\mathrm{H} \cdots \mathrm{O}, \mathrm{O}-\mathrm{H} \cdots \mathrm{O}, \mathrm{N}-\mathrm{H} \cdots(\mathrm{O}, \mathrm{O})$ and $\mathrm{O}-\mathrm{H} \cdots(\mathrm{O}, \mathrm{O})$ hydrogen bonds, generating a three-dimensional network. Weak $\mathrm{C}-\mathrm{H} \cdots \mathrm{O}$ interactions consolidate the packing.

\section{Chemical context}

Polyoxometalates (POMs) are clusters of transition metals ( $M$ $=\mathrm{V}, \mathrm{Nb}, \mathrm{Ta}, \mathrm{Mo}, \mathrm{W}, \cdots)$ and oxygen atoms with a structural and compositional diversity that lead to numerous applications because of their electrochemical, optical, catalytic and photochromic properties as well as their antiviral and antitumor activities (Katsoulis, 1998; Hasenknopf, 2005; Gerth et al., 2005; Coué et al., 2007). In this context, the $\left[\mathrm{Mo}_{7} \mathrm{O}_{24}\right]^{6-}$ heptamolybdate anion has been isolated with numerous different counter-cations such as 4-aminopyridinium, $N$ pentylammonium, diethylenetriammonium, $N, N, N^{\prime}, N^{\prime}$-tetramethylethylenediammonium, tetramethylammonium, guanidinium, hexanediammonium, butan-1-aminium, ammonium, potassium and sodium (Román et al., 1985, 1988, 1990, 1992; Don \& Weakly, 1981; Gatehouse \& Leverett, 1968; Sjöbom \& Hedman, 1973; Niu et al., 1996; Himeno et al., 1997; Reinoso et al., 2008; Ftini, 2015; Khandolkar et al., 2016). As a continuation of our work in this area (Sarr et al., 2018), we now describe the synthesis and structure of the title compound (I), which is notable for the incorporation of both protonated $\left[\mathrm{C}_{4} \mathrm{H}_{7} \mathrm{~N}_{2}\right]^{+}$2-methylimidazolium cations and neutral $\mathrm{C}_{4} \mathrm{H}_{6} \mathrm{~N}_{2}$ 2-methylimidazole molecules in the crystal.

\section{Structural commentary}

The title compound is characterized by the presence of the familiar $\left[\mathrm{Mo}_{7} \mathrm{O}_{24}\right]^{6-}$ heptamolybdate cluster anion (Fig. 1). 


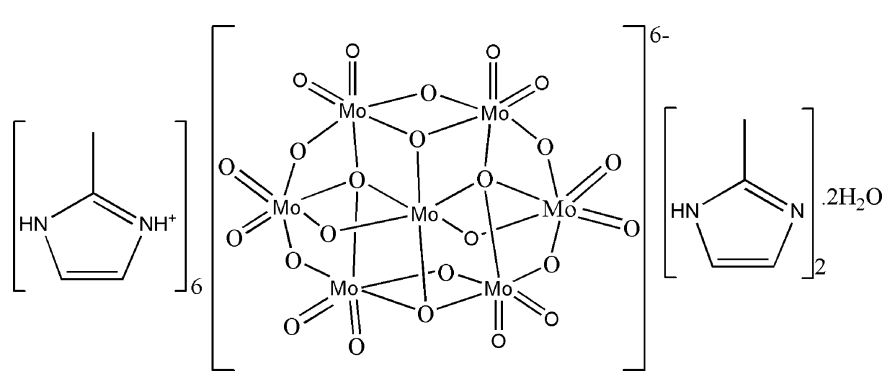

There are four categories of oxygen atoms within the polyanion: $\mathrm{O}_{\mathrm{t}}$ (terminal oxygen atoms), $\mu^{2}-\mathrm{O}$ (oxygen atoms bridging two molybdenum atoms), $\mu^{3}-\mathrm{O}$ (oxygen atoms bridging three molybdenum atoms) and $\mu^{4}-\mathrm{O}$ (oxygen atoms bridging four molybdenum atoms). All of the Mo atoms are bound to two terminal oxygen atoms except for Mo7, which is located in the 'core' of the cluster. The geometrical data for the cluster in (I) are consistent with those found in previous studies (Román et al., 1992; Reinoso et al., 2008): the Mo-O bond lengths vary between 1.707 (2) and 1.726 (2) $\AA$ for $\mathrm{O}_{\mathrm{t}}$, 1.754 (2)-2.453 (2) $\AA$ for $\mu^{2}-\mathrm{O}, 1.8945$ (19)-2.3057 (19) $\AA$ for $\mu^{3}$-O and $2.1329(19)-2.3011$ (18) $\AA$ for $\mu^{4}$-O. The variations of $\mathrm{Mo}-\mathrm{O}$ bond lengths and $\mathrm{O}-\mathrm{Mo}-\mathrm{O}$ angles indicate that all seven octahedra $\left(\mathrm{MoO}_{6}\right)$ within the cluster are highly distorted. As in the compound $\left(\mathrm{H}_{3} \mathrm{dien}\right)_{2}\left[\mathrm{Mo}_{7} \mathrm{O}_{24}\right] \cdot 4 \mathrm{H}_{2} \mathrm{O}$ (Román et al., 1988), we note that the longest Mo-O bond length derives from an oxygen atom bridging two molybdenum atoms $\left(\mu^{2}-\mathrm{O}\right)$. As well as the $\left[\mathrm{Mo}_{7} \mathrm{O}_{24}\right]^{6-}$ anion, six $\left(\mathrm{C}_{4} \mathrm{H}_{7} \mathrm{~N}_{2}\right)^{+}$cations, two neutral $\mathrm{C}_{4} \mathrm{H}_{6} \mathrm{~N}_{2}$ molecules and two water molecules of crystallization are present in the asymmetric unit (Fig. 2).

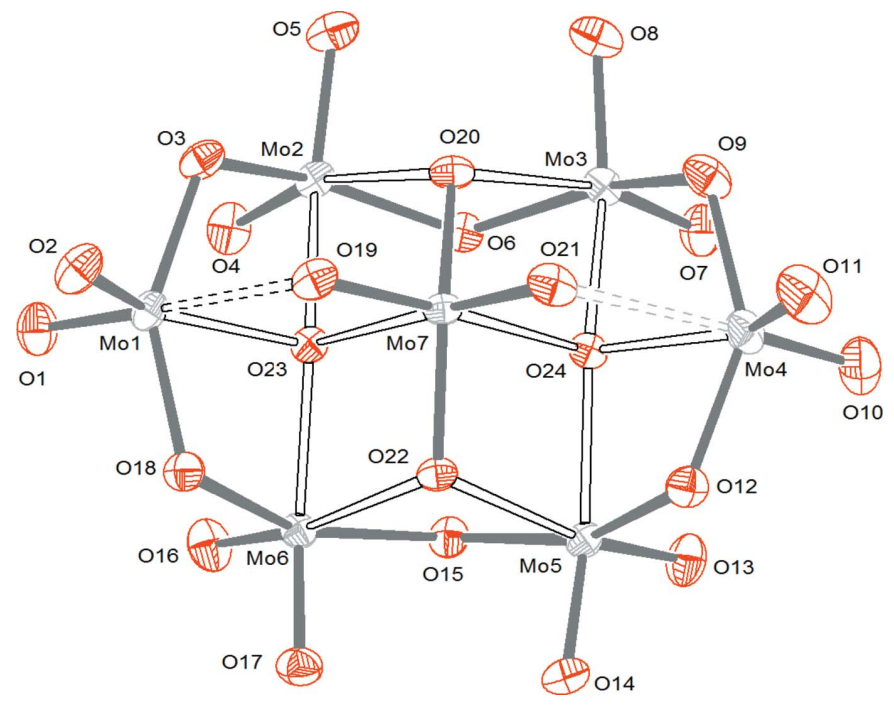

Figure 1

Molecular structure of the $\left[\mathrm{Mo}_{7} \mathrm{O}_{24}\right]^{6-}$ heptamolybdate cluster anion in (I).

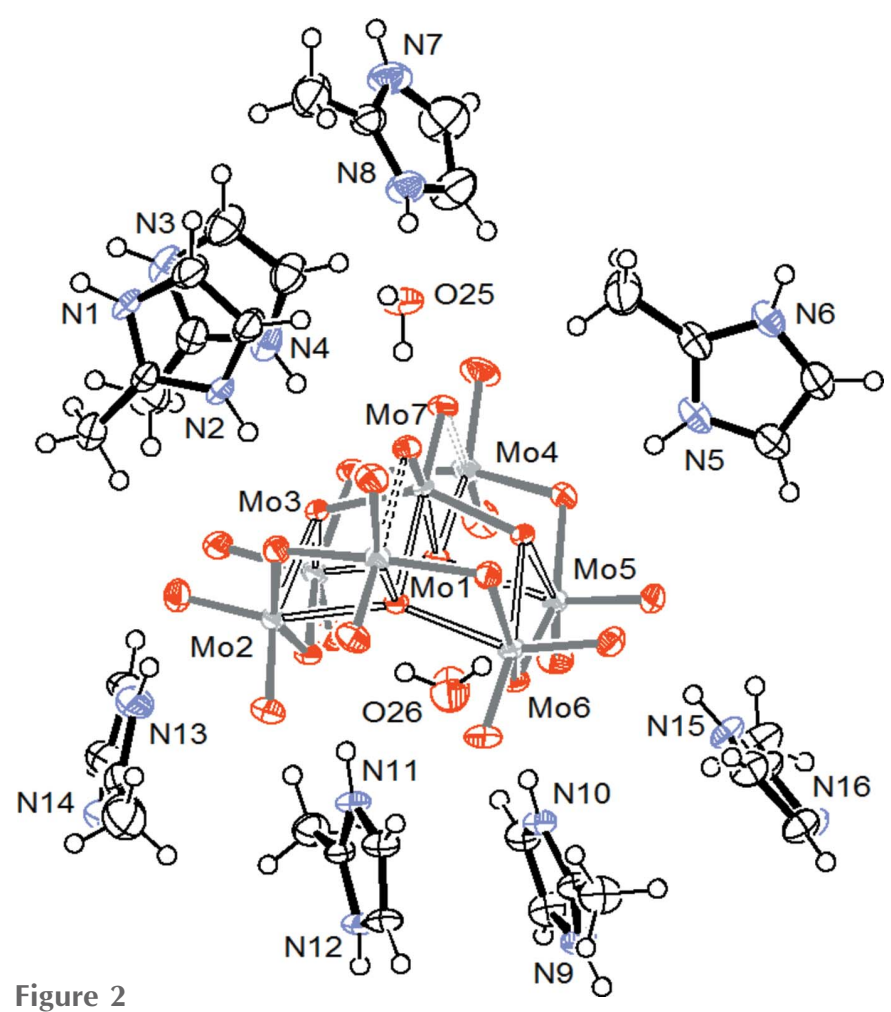

The asymmetric unit of (I) with displacement ellipsoids drawn at the $50 \%$ probability level.

\section{Supramolecular features}

In the crystal, each heptamolybdate anion interacts with six neighbours via the water molecules, $\left(\mathrm{C}_{4} \mathrm{H}_{7} \mathrm{~N}_{2}\right)^{+}$cations and/or neutral 2-methylimidazole molecules (Fig. 3). These interactions occur through simple $\mathrm{O}-\mathrm{H} \cdots \mathrm{O}, \mathrm{N}-\mathrm{H} \cdots \mathrm{O}$ and $\mathrm{N}-$ $\mathrm{H} \cdots \mathrm{N}$ and bifurcated $\mathrm{N}-\mathrm{H} \cdots(\mathrm{O}, \mathrm{O})$ and $\mathrm{O}-\mathrm{H} \cdots(\mathrm{O}, \mathrm{O})$

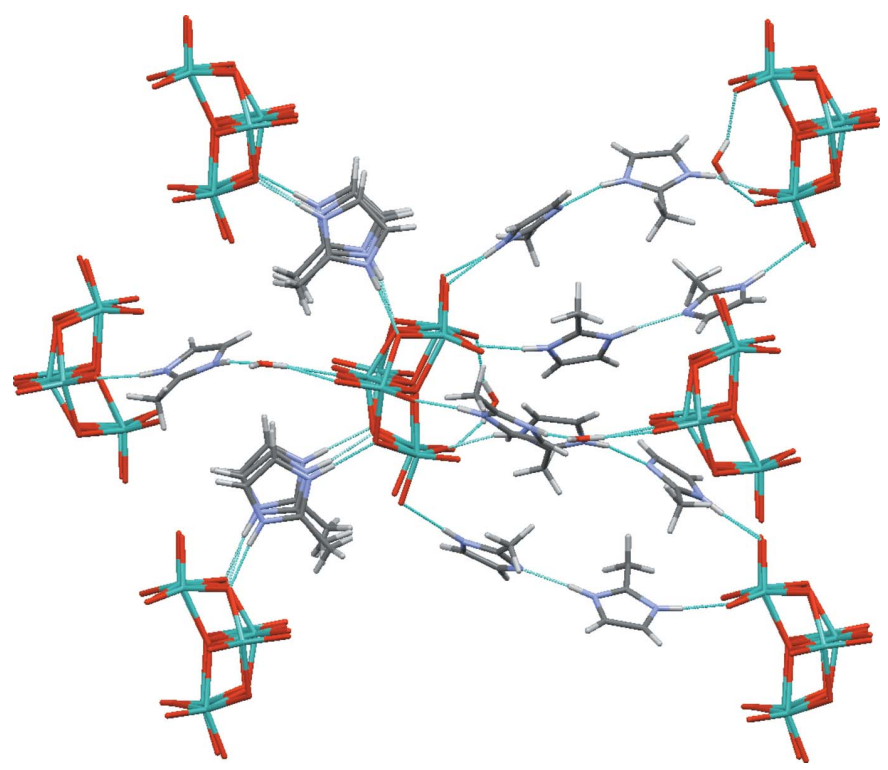

Figure 3

Detail of the structure of (I) showing the interconnections of the $\left[\mathrm{Mo}_{7} \mathrm{O}_{24}\right]^{6-}$ anion with its neighbours. 
Table 1

Hydrogen-bond geometry $\left(\AA,^{\circ}\right)$.

\begin{tabular}{lllll}
\hline$D-\mathrm{H} \cdots A$ & $D-\mathrm{H}$ & $\mathrm{H} \cdots A$ & $D \cdots A$ & $D-\mathrm{H} \cdots A$ \\
\hline $\mathrm{N} 1-\mathrm{H} 1 N \cdots \mathrm{O} 22^{\mathrm{i}}$ & $0.88(2)$ & $1.85(2)$ & $2.710(3)$ & $167(2)$ \\
$\mathrm{N} 2-\mathrm{H} 2 N \cdots \mathrm{O} 20$ & $0.87(2)$ & $1.79(2)$ & $2.655(3)$ & $172(2)$ \\
$\mathrm{N} 3-\mathrm{H} 3 N \cdots \mathrm{O} 18^{\mathrm{i}}$ & $0.88(2)$ & $1.87(2)$ & $2.743(4)$ & $171(5)$ \\
$\mathrm{N} 4-\mathrm{H} 4 N \cdots \mathrm{O} 9$ & $0.88(2)$ & $1.79(2)$ & $2.662(4)$ & $171(5)$ \\
$\mathrm{N} 5-\mathrm{H} 5 N \cdots \mathrm{O} 12$ & $0.89(2)$ & $1.77(3)$ & $2.644(4)$ & $168(4)$ \\
$\mathrm{N} 6-\mathrm{H} 6 N \cdots \mathrm{O} 3^{\text {ii }}$ & $0.90(2)$ & $1.80(3)$ & $2.684(4)$ & $168(5)$ \\
$\mathrm{N} 7-\mathrm{H} 7 N \cdots \mathrm{O} 1^{\text {iii }}$ & $0.89(3)$ & $1.83(3)$ & $2.697(4)$ & $167(3)$ \\
$\mathrm{N} 8-\mathrm{H} 8 N \cdots \mathrm{O} 25$ & $0.89(3)$ & $1.78(3)$ & $2.659(4)$ & $172(5)$ \\
$\mathrm{N} 9-\mathrm{H} 9 N \cdots \mathrm{N} 14^{\text {iv }}$ & $0.89(2)$ & $1.81(2)$ & $2.698(4)$ & $177(3)$ \\
$\mathrm{N} 10-\mathrm{H} 10 N \cdots \mathrm{O} 15$ & $0.88(2)$ & $1.98(2)$ & $2.852(3)$ & $174(3)$ \\
$\mathrm{N} 11-\mathrm{H} 11 N \cdots \mathrm{O} 6$ & $0.88(2)$ & $1.78(2)$ & $2.636(3)$ & $166(3)$ \\
$\mathrm{N} 12-\mathrm{H} 12 N \cdots \mathrm{N} 16^{\mathrm{v}}$ & $0.88(2)$ & $1.87(2)$ & $2.725(4)$ & $165(3)$ \\
$\mathrm{N} 13-\mathrm{H} 13 N \cdots \mathrm{O} 5$ & $0.88(4)$ & $2.32(4)$ & $3.186(5)$ & $167(3)$ \\
$\mathrm{N} 13-\mathrm{H} 13 N \cdots \mathrm{O} 8$ & $0.88(4)$ & $2.55(4)$ & $3.043(5)$ & $116(2)$ \\
$\mathrm{N} 15-\mathrm{H} 15 N \cdots \mathrm{O} 14$ & $0.87(3)$ & $2.22(3)$ & $2.953(4)$ & $142(3)$ \\
$\mathrm{N} 15-\mathrm{H} 15 N \cdots \mathrm{O} 17$ & $0.87(3)$ & $2.27(3)$ & $2.917(4)$ & $132(3)$ \\
$\mathrm{O} 25-\mathrm{H} 25 V \cdots \mathrm{O} 19$ & $0.86(2)$ & $2.00(2)$ & $2.788(3)$ & $154(5)$ \\
$\mathrm{O} 25-\mathrm{H} 25 W \cdots \mathrm{O} 26^{\text {vi }}$ & $0.86(3)$ & $1.85(3)$ & $2.702(4)$ & $176(5)$ \\
$\mathrm{O} 26-\mathrm{H} 26 V \cdots \mathrm{O} 10$ & $0.83(4)$ & $2.50(6)$ & $2.999(4)$ & $119(5)$ \\
$\mathrm{O} 26-\mathrm{H} 26 V \cdots \mathrm{O} 13$ & $0.83(4)$ & $2.25(5)$ & $3.009(4)$ & $151(6)$ \\
$\mathrm{O} 26-\mathrm{H} 26 W \cdots \mathrm{O} 7$ & $0.83(4)$ & $1.99(6)$ & $2.737(4)$ & $149(6)$ \\
$\mathrm{C} 4-\mathrm{H} 4 A \cdots \mathrm{O} 8$ & 0.98 & 2.35 & $3.228(5)$ & 149 \\
$\mathrm{C} 8-\mathrm{H} 8 B \cdots \mathrm{O} 2^{\mathrm{i}}$ & 0.98 & 2.54 & $3.517(6)$ & 176 \\
$\mathrm{C} 8-\mathrm{H} 8 C \cdots \mathrm{O} 8$ & 0.98 & 2.49 & $3.386(5)$ & 152 \\
$\mathrm{C} 10-\mathrm{H} 10 \cdots \mathrm{O} 26^{\text {vii }}$ & 0.95 & 2.45 & $3.318(5)$ & 151 \\
$\mathrm{C} 12-\mathrm{H} 12 A \cdots \mathrm{O} 5^{\text {ii }}$ & 0.98 & 2.44 & $3.407(5)$ & 167 \\
$\mathrm{C} 13-\mathrm{H} 13 \cdots \mathrm{O} 4^{\mathrm{iii}}$ & 0.95 & 2.50 & $3.297(6)$ & 141 \\
$\mathrm{C} 17-\mathrm{H} 17 \cdots \mathrm{O} 2^{\text {viii }}$ & 0.95 & 2.46 & $3.154(4)$ & 130 \\
$\mathrm{C} 18-\mathrm{H} 18 \cdots \mathrm{O} 26$ & 0.95 & 2.58 & $3.522(5)$ & 172 \\
$\mathrm{C} 21-\mathrm{H} 21 \cdots \mathrm{O} 16$ & 0.95 & 2.46 & $3.302(4)$ & 147 \\
$\mathrm{C} 22-\mathrm{H} 22 \cdots \mathrm{O} 11^{\mathrm{ix}}$ & 0.95 & 2.22 & $3.113(4)$ & 156 \\
$\mathrm{C} 24-\mathrm{H} 24 B \cdots \mathrm{O} 7$ & 0.98 & 2.50 & $3.346(5)$ & 145 \\
$\mathrm{C} 25-\mathrm{H} 25 \cdots \mathrm{O} 8$ & 0.95 & 2.59 & $3.055(5)$ & 110 \\
$\mathrm{C} 32-\mathrm{H} 32 B \cdots \mathrm{O} 14$ & 0.98 & 2.55 & $3.234(5)$ & 126 \\
\hline & & & &
\end{tabular}

Symmetry codes: (i) $\quad-x+\frac{1}{2}, y-\frac{1}{2},-z+\frac{1}{2}$; $\quad$ (ii) $\quad-x+\frac{1}{2}, y+\frac{1}{2},-z+\frac{1}{2}$; $x+\frac{1}{2},-y+\frac{1}{2}, z-\frac{1}{2} ; \quad$ (iv) $\quad-x+\frac{1}{2}, y+\frac{1}{2},-z+\frac{3}{2}$; $\quad$ (v) $-x+\frac{1}{2}, y-\frac{1}{2},-z+\frac{3}{2}$; $\quad$ (vi) $x-\frac{1}{2},-y+\frac{1}{2}, z-\frac{1}{2} ; \quad$ (vii) $-x+1,-y+1,-z+1$; (viii) $x+\frac{1}{2},-y+\frac{1}{2}, z+\frac{1}{2}$; (ix) $x-\frac{1}{2},-y+\frac{1}{2}, z+\frac{1}{2}$

hydrogen bonds (Table 1) involving three categories of oxygen atoms of the polyanion: $\mathrm{O}_{\mathrm{t}}, \mu^{2}-\mathrm{O}$ and $\mu^{3}-\mathrm{O}$. The $\mathrm{N}-\mathrm{H} \cdots \mathrm{N}$ hydrogen bonds from $\mathrm{N} 9$ and $\mathrm{N} 12$ link $\left(\mathrm{C}_{4} \mathrm{H}_{7} \mathrm{~N}_{2}\right)^{+}$cations to neutral molecules. The packing is consolidated by weak $\mathrm{C}-$ $\mathrm{H}$... O links (Table 1). The overall hydrogen-bonding topology is an infinite three-dimensional network.

\section{Database survey}

A search of the Cambridge Structural Database (CSD, version 5.40, update November 2018; Groom et al., 2016) resulted in 35 hits for the heptamolybdate anion and 90 hits for the 2-methylimidazolium cation.

\section{Synthesis and crystallization}

Sulfuric acid $(2.1 \mathrm{~g}, 21.7 \mathrm{mmol}), 2$-methylimidazole $(3.5 \mathrm{~g}$, $43.4 \mathrm{mmol}$ ) and ammonium heptamolybdate tetrahydrate $(2.2 \mathrm{~g}, 1.8 \mathrm{mmol})$ in a ratio of 1:2:1/12 were dissolved in water $(60 \mathrm{ml})$. The solution was stirred for one $\mathrm{h}$ and evaporated in the oven at $333 \mathrm{~K}$ to yield a whitish precipitate. The precipitate was recrystallized from methanol solution: after two
Table 2

Experimental details.

\begin{tabular}{|c|c|}
\hline \multicolumn{2}{|l|}{ Crystal data } \\
\hline Chemical formula & $\begin{array}{l}\left(\mathrm{C}_{4} \mathrm{H}_{7} \mathrm{~N}_{2}\right)_{6}\left[\mathrm{Mo}_{7} \mathrm{O}_{24}\right] \cdot 2 \mathrm{C}_{4} \mathrm{H}_{6} \mathrm{~N}_{2} \cdot- \\
\quad 2 \mathrm{H}_{2} \mathrm{O}\end{array}$ \\
\hline$M_{\mathrm{r}}$ & 1754.52 \\
\hline Crystal system, space group & Monoclinic, $P 2_{1} / n$ \\
\hline Temperature (K) & 170 \\
\hline$a, b, c(\AA)$ & $\begin{array}{l}16.5325(2), 17.5842(2), \\
19.8873(2)\end{array}$ \\
\hline$\beta\left({ }^{\circ}\right)$ & $90.653(1)$ \\
\hline$V\left(\AA^{6}\right)$ & $5781.08(11)$ \\
\hline$Z$ & 4 \\
\hline Radiation type & Мo $K \alpha$ \\
\hline$\mu\left(\mathrm{mm}^{-1}\right)$ & 1.56 \\
\hline Crystal size $(\mathrm{mm})$ & $0.38 \times 0.28 \times 0.19$ \\
\hline \multicolumn{2}{|l|}{ Data collection } \\
\hline Diffractometer & Agilent Xcalibur, Sapphire2 \\
\hline Absorption correction & $\begin{array}{l}\text { Multi-scan (CrysAlis PRO; } \\
\text { Agilent, 2014) }\end{array}$ \\
\hline$T_{\min }, T_{\max }$ & $0.476,0.756$ \\
\hline $\begin{array}{l}\text { No. of measured, independent and } \\
\text { observed }[I>2 \sigma(I)] \text { reflections }\end{array}$ & $104921,27993,21593$ \\
\hline$R_{\text {int }}$ & 0.053 \\
\hline$(\sin \theta / \lambda)_{\max }\left(\AA^{-1}\right)$ & 0.833 \\
\hline \multicolumn{2}{|l|}{ Refinement } \\
\hline$R\left[F^{2}>2 \sigma\left(F^{2}\right)\right], w R\left(F^{2}\right), S$ & $0.046,0.120,1.15$ \\
\hline No. of reflections & 27993 \\
\hline No. of parameters & 792 \\
\hline No. of restraints & 58 \\
\hline H-atom treatment & $\begin{array}{l}\mathrm{H} \text { atoms treated by a mixture of } \\
\text { independent and constrained } \\
\text { refinement }\end{array}$ \\
\hline$\Delta \rho_{\max }, \Delta \rho_{\min }\left(\mathrm{e} \AA^{-3}\right)$ & $1.83,-2.87$ \\
\hline
\end{tabular}

Computer programs: CrysAlis PRO (Agilent, 2014), SIR92 (Altomare et al., 1992), SHELXL97 (Sheldrick, 2008), ORTEP-3 for Windows and WinGX (Farrugia, 2012), Mercury (Macrae et al., 2008) and PLATON (Spek, 2009).

weeks at room temperature, colourless prisms of (I) were recovered.

The IR spectrum of (I) is included in the supporting information. The absorption bands at 3400 and $3395 \mathrm{~cm}^{-1}$ correspond to $v(\mathrm{O}-\mathrm{H})$ stretches and indicate the presence of water molecules and those at 1621 and $1564 \mathrm{~cm}^{-1}$ to the deformation vibrations $\delta(\mathrm{O}-\mathrm{H})$. The bands centered at 3132 and $1431 \mathrm{~cm}^{-1}$ with shoulders are respectively attributed to the stretching and deformation vibrations of the $\mathrm{N}-\mathrm{H}$ bonds of the protonated and/or non-protonated entities of 2-methylimidazole (Jinnah et al., 2004). The bands between 2904$2686 \mathrm{~cm}^{-1}$ are attributed to the stretching vibrations of the $\mathrm{C}-\mathrm{H}$ bonds, while that at $1291 \mathrm{~cm}^{-1}$ is a $\delta(\mathrm{C}-\mathrm{H})$ deformation vibration (Jinnah et al., 2004). The two bands at 929 and $900 \mathrm{~cm}^{-1}$ correspond to $v(\mathrm{Mo}-\mathrm{Ot})$ stretching vibrations while the bands between 838 and $650 \mathrm{~cm}^{-1}$ are typical for the vibrations of $v(\mathrm{Mo}-\mathrm{O}-\mathrm{Mo})$ and $v[\mathrm{Mo}-(\mu-\mathrm{O})]$ groupings (Dey et al., 2011).

\section{Refinement details}

Crystal data, data collection and structure refinement details are summarized in Table 2. All $\mathrm{H}$ atoms treated by a mixture of independent and constrained refinement were placed in 
geometrically idealized positions and constrained to ride on their parent atoms, with $\mathrm{N}-\mathrm{H}$ distances of 0.87 (2), 0.88 (2) and 0.89 (2) $\AA$, Cmethyl $-\mathrm{H}=0.97 / 0.98 \AA$ and Cmethine $-\mathrm{H}$ $=0.94 / 0.95 \AA$, and with $U_{\text {iso }}(\mathrm{H})=1.2 U_{\text {eq }}(\mathrm{C}, \mathrm{N})$ or $1.5 U_{\text {eq }}(\mathrm{C}-$ methyl).

\section{Acknowledgements}

The authors thank the Université Cheikh Anta Diop DakarSénégal, the Laboratoire de Chimie et de Physique des Matériaux (LCPM) de l'Université Assane Seck de Ziguinchor, Sénégal and the service commun d'analyse par diffraction des rayons $X$, Universitée de Bretagne Occidentale, France for financial support. All measurements were performed in the institutes above quoted.

\section{References}

Agilent (2014). CrysAlis PRO. Agilent Technologies, Yarnton, England.

Altomare, A., Cascarano, G., Giacovazzo, C., Guagliardi, A., Burla, M. C., Polidori, G. \& Camalli, M. (1992). J. Appl. Cryst. 27, 435.

Coué, V., Dessapt, R., Bujoli-Doeuff, M., Evain, M. \& Jobic, S. (2007). Inorg. Chem. 46, 2824-2835.

Dey, C. D., Das, R., Pachfule, P., Poddar, P. \& Banerjee, R. (2011). Cryst. Growth Des. 11, 139-146.

Don, A. \& Weakley, T. J. R. (1981). Acta Cryst. B37, 451-453.

Farrugia, L. J. (2012). J. Appl. Cryst. 45, 849-854.

Ftini, M. M. (2015). J. Struct. Chem. 56, 1595-1601.

Gatehouse, B. M. \& Leverett, P. (1968). Chem. Commun. pp. 901902.
Gerth, H. U. V., Rompel, A., Krebs, B., Boos, J. \& Lanvers-Kaminsky, C. (2005). Anticancer Drugs, 16, 101-106.

Groom, C. R., Bruno, I. J., Lightfoot, M. P. \& Ward, S. C. (2016). Acta Cryst. B72, 171-179.

Hasenknopf, B. (2005). Front. Biosci. 10, 275-287.

Himeno, S., Niiya, H. \& Ueda, T. (1997). Bull. Chem. Soc. Jpn, 70 , 631-637.

Jinnah, M. M. A., Umadevi, M. \& Ramakrishnan, V. (2004). J. Raman Spectrosc. 35, 956-960.

Katsoulis, D. E. (1998). Chem. Rev. 98, 359-388.

Khandolkar, S. S., Naik, A. R., Näther, C., Bensch, W. \& Srinivasan, B. R. (2016). J. Chem. Sci. 128, 1737-1744.

Macrae, C. F., Bruno, I. J., Chisholm, J. A., Edgington, P. R., McCabe, P., Pidcock, E., Rodriguez-Monge, L., Taylor, R., van de Streek, J. \& Wood, P. A. (2008). J. Appl. Cryst. 41, 466-470.

Niu, J.-Y., You, X.-Z., Fun, H.-K., Zhou, Z.-Y. \& Yip, B.-C. (1996). Polyhedron, 15, 1003-1008.

Reinoso, S., Dickman, M. H., Praetorius, A. \& Kortz, U. (2008). Acta Cryst. E64, m614-m615.

Román, P., Gutiérrez-Zorrilla, J. M., Luque, A. \& Martínez-Ripoll, M. (1988). J. Crystallogr. Spectrosc. Res. 18, 117-131.

Román, P., Gutiérrez-Zorrilla, J. M., Martínez-Ripoll, M. \& GarcíaBlanco, S. (1985). Z. Kristallogr. 173, 283-292.

Román, P., Luque, A., Aranzabe, A. \& Gutiérrez-Zorrilla, J. M. (1992). Polyhedron, 11, 2027-2038.

Román, P., Luque, A., Gutiérrez-Zorrilla, J. M. \& Zuniga, F. J. (1990). Z. Kristallogr. 190, 249-258.

Sarr, B., Diop, C. A. K., Melin, F., Sidibe, M., Hellwig, P., Michaud, F., Maury, F., Senocq, F., Mbaye, A. \& Rousselin, Y. (2018). J. Mol. Struct. 1170, 44-50.

Sheldrick, G. M. (2008). Acta Cryst. A64, 112-122.

Sjöbom, K. \& Hedman, B. (1973). Acta Chem. Scand. A27, 36733691.

Spek, A. L. (2009). Acta Cryst. D65, 148-155. 


\section{supporting information}

Acta Cryst. (2019). E75, 1001-1004 [https://doi.org/10.1107/S2056989019008454]

Synthesis and crystal structure of a new hybrid organic-inorganic material containing neutral molecules, cations and heptamolybdate anions

Bougar Sarr, Abdou Mbaye, Wally Diallo, Cheikh Abdoul Khadir Diop, Mamadou Sidibe and Francois Michaud

Computing details

Data collection: CrysAlis PRO (Agilent, 2014); cell refinement: CrysAlis PRO (Agilent, 2014); data reduction: CrysAlis PRO (Agilent, 2014); program(s) used to solve structure: SIR92 (Altomare et al., 1992); program(s) used to refine structure: SHELXL97 (Sheldrick, 2008); molecular graphics: ORTEP-3 for Windows (Farrugia, 2012) and Mercury (Macrae et al., 2008); software used to prepare material for publication: WinGX (Farrugia, 2012) and PLATON (Spek, 2009).

Hexakis(2-methyl-1H-imidazol-3-ium) heptamolybdate 2-methyl-1H-imidazole disolvate dihydrate

Crystal data

$\left(\mathrm{C}_{4} \mathrm{H}_{7} \mathrm{~N}_{2}\right)_{6}\left[\mathrm{Mo}_{7} \mathrm{O}_{24}\right] \cdot 2 \mathrm{C}_{4} \mathrm{H}_{6} \mathrm{~N}_{2} \cdot 2 \mathrm{H}_{2} \mathrm{O}$

$F(000)=3456$

$M_{r}=1754.52$

Monoclinic, $P 2_{1} / n$

Hall symbol: -P 2 yn

$a=16.5325$ (2) $\AA$

$b=17.5842(2) \AA$

$c=19.8873(2) \AA$

$D_{\mathrm{x}}=2.016 \mathrm{Mg} \mathrm{m}^{-3}$

Mo $K \alpha$ radiation, $\lambda=0.71073 \AA$

Cell parameters from 38522 reflections

$\theta=3.5-37.4^{\circ}$

$\mu=1.56 \mathrm{~mm}^{-1}$

$T=170 \mathrm{~K}$

$\beta=90.653(1)^{\circ}$

Fragment of prism, colourless

$V=5781.08(11) \AA^{3}$

$0.38 \times 0.28 \times 0.19 \mathrm{~mm}$

$Z=4$

Data collection

Agilent Xcalibur, Sapphire2, large Be window diffractometer

Radiation source: Enhance (Mo) X-ray Source Graphite monochromator

Detector resolution: 8.3622 pixels $\mathrm{mm}^{-1}$

$\omega$ scans

Absorption correction: multi-scan

(CrysAlis PRO; Agilent, 2014)

$T_{\text {min }}=0.476, T_{\max }=0.756$

104921 measured reflections 27993 independent reflections 21593 reflections with $I>2 \sigma(I)$

$R_{\text {int }}=0.053$

$\theta_{\text {max }}=36.3^{\circ}, \theta_{\min }=3.3^{\circ}$

$h=-24 \rightarrow 27$

$k=-29 \rightarrow 29$

$l=-33 \rightarrow 33$

Refinement

Refinement on $F^{2}$

Least-squares matrix: full

27993 reflections

$R\left[F^{2}>2 \sigma\left(F^{2}\right)\right]=0.046$

792 parameters

$w R\left(F^{2}\right)=0.120$

$S=1.15$

58 restraints

Primary atom site location: structure-invariant direct methods 
Secondary atom site location: difference Fourier map

Hydrogen site location: inferred from neighbouring sites

$\mathrm{H}$ atoms treated by a mixture of independent and constrained refinement

$$
\begin{aligned}
& w=1 /\left[\sigma^{2}\left(F_{\mathrm{o}}^{2}\right)+(0.0397 P)^{2}+11.2859 P\right] \\
& \text { where } P=\left(F_{\mathrm{o}}^{2}+2 F_{\mathrm{c}}^{2}\right) / 3 \\
& (\Delta / \sigma)_{\max }=0.011 \\
& \Delta \rho_{\max }=1.83 \text { e } \AA^{-3} \\
& \Delta \rho_{\min }=-2.87 \mathrm{e} \AA^{-3}
\end{aligned}
$$

\section{Special details}

Geometry. All s.u.'s (except the s.u. in the dihedral angle between two l.s. planes) are estimated using the full covariance matrix. The cell s.u.'s are taken into account individually in the estimation of s.u.'s in distances, angles and torsion angles; correlations between s.u.'s in cell parameters are only used when they are defined by crystal symmetry. An approximate (isotropic) treatment of cell s.u.'s is used for estimating s.u.'s involving 1.s. planes.

Refinement. Refinement of $\mathrm{F}^{2}$ against ALL reflections. The weighted R-factor $\mathrm{wR}$ and goodness of fit $\mathrm{S}$ are based on $\mathrm{F}^{2}$,

\begin{tabular}{|c|c|c|c|c|}
\hline & $x$ & $y$ & $z$ & $U_{\text {iso }} * / U_{\text {eq }}$ \\
\hline O1 & $-0.04305(13)$ & $0.24793(14)$ & $0.39565(12)$ & $0.0280(4)$ \\
\hline $\mathrm{O} 2$ & $0.00551(14)$ & $0.27946(14)$ & $0.26843(11)$ & $0.0284(5)$ \\
\hline $\mathrm{O} 3$ & $0.07596(13)$ & $0.15830(12)$ & $0.34241(10)$ & $0.0230(4)$ \\
\hline $\mathrm{O} 4$ & $0.07336(14)$ & $0.12579(14)$ & $0.48407(11)$ & $0.0269(4)$ \\
\hline O5 & $0.15457(15)$ & $0.03530(13)$ & $0.39571(12)$ & $0.0295(5)$ \\
\hline O6 & $0.23807(12)$ & $0.13563(11)$ & $0.47918(9)$ & $0.0185(3)$ \\
\hline $\mathrm{O} 7$ & $0.40034(14)$ & $0.12283(14)$ & $0.50582(12)$ & $0.0284(5)$ \\
\hline O8 & $0.33831(15)$ & $0.04054(13)$ & $0.40157(12)$ & $0.0280(4)$ \\
\hline O9 & $0.42573(14)$ & $0.16391(13)$ & $0.36926(12)$ & $0.0268(4)$ \\
\hline $\mathrm{O} 10$ & $0.52563(15)$ & $0.25984(16)$ & $0.44633(15)$ & $0.0364(6)$ \\
\hline O11 & $0.50709(16)$ & 0.28585 (17) & $0.31122(14)$ & $0.0393(6)$ \\
\hline $\mathrm{O} 12$ & $0.41549(13)$ & $0.37036(12)$ & $0.40412(11)$ & $0.0223(4)$ \\
\hline $\mathrm{O} 13$ & $0.39387(13)$ & $0.34235(15)$ & $0.54308(11)$ & $0.0277(4)$ \\
\hline O14 & $0.32385(14)$ & $0.46326(12)$ & $0.47845(11)$ & $0.0246(4)$ \\
\hline $\mathrm{O} 15$ & $0.23109(12)$ & $0.33656(12)$ & $0.51719(9)$ & $0.0197(3)$ \\
\hline O16 & $0.06318(13)$ & $0.33595(15)$ & $0.52098(11)$ & $0.0278(5)$ \\
\hline $\mathrm{O} 17$ & $0.13927(14)$ & $0.45859(13)$ & $0.46625(11)$ & $0.0251(4)$ \\
\hline $\mathrm{O} 18$ & $0.06704(13)$ & $0.36491(12)$ & $0.37905(10)$ & $0.0213(4)$ \\
\hline O19 & $0.16800(13)$ & $0.28094(12)$ & $0.28601(10)$ & $0.0227(4)$ \\
\hline $\mathrm{O} 20$ & $0.24914(12)$ & $0.16416(11)$ & $0.35550(9)$ & $0.0191(3)$ \\
\hline $\mathrm{O} 21$ & $0.33322(14)$ & $0.28445(13)$ & $0.29412(11)$ & $0.0253(4)$ \\
\hline $\mathrm{O} 22$ & $0.24226(12)$ & $0.36427(11)$ & $0.39099(9)$ & $0.0171(3)$ \\
\hline $\mathrm{O} 23$ & $0.14447(11)$ & $0.25197(11)$ & $0.41666(9)$ & $0.0164(3)$ \\
\hline $\mathrm{O} 24$ & $0.33781(11)$ & $0.25392(11)$ & $0.42940(9)$ & $0.0172(3)$ \\
\hline Mo1 & $0.042356(14)$ & $0.264056(14)$ & $0.348758(11)$ & $0.01907(5)$ \\
\hline Mo2 & $0.142420(14)$ & $0.128403(13)$ & $0.420960(11)$ & $0.01805(4)$ \\
\hline Mo3 & $0.342279(14)$ & $0.130828(14)$ & $0.434127(12)$ & $0.01888(4)$ \\
\hline Mo4 & $0.454713(15)$ & $0.269540(15)$ & $0.383726(13)$ & $0.02328(5)$ \\
\hline Mo5 & $0.333156(14)$ & $0.365314(13)$ & $0.475739(11)$ & $0.01745(4)$ \\
\hline
\end{tabular}
conventional R-factors $\mathrm{R}$ are based on $\mathrm{F}$, with $\mathrm{F}$ set to zero for negative $\mathrm{F}^{2}$. The threshold expression of $\mathrm{F}^{2}>2 \sigma\left(\mathrm{F}^{2}\right)$ is used only for calculating R-factors(gt) etc. and is not relevant to the choice of reflections for refinement. R-factors based on $\mathrm{F}^{2}$ are statistically about twice as large as those based on $\mathrm{F}$, and R- factors based on ALL data will be even larger.

Fractional atomic coordinates and isotropic or equivalent isotropic displacement parameters $\left(\AA^{2}\right)$ 


\begin{tabular}{|c|c|c|c|c|}
\hline Mo6 & $0.134696(14)$ & $0.360541(13)$ & $0.463691(11)$ & $0.01669(4)$ \\
\hline Mo7 & $0.248954(14)$ & $0.271359(12)$ & $0.343147(10)$ & $0.01554(4)$ \\
\hline $\mathrm{C} 1$ & $0.2405(3)$ & $0.0776(2)$ & $0.1309(2)$ & $0.0436(9)$ \\
\hline H1 & 0.235 & 0.0891 & 0.0844 & $0.052 *$ \\
\hline $\mathrm{C} 2$ & $0.2374(3)$ & $0.1281(2)$ & $0.18283(19)$ & $0.0366(8)$ \\
\hline $\mathrm{H} 2$ & 0.2294 & 0.1814 & 0.1794 & $0.044^{*}$ \\
\hline $\mathrm{C} 3$ & $0.25571(18)$ & $0.01393(18)$ & $0.22512(16)$ & $0.0243(5)$ \\
\hline $\mathrm{C} 4$ & $0.2682(3)$ & -0.0505 (2) & $0.2726(2)$ & $0.0466(10)$ \\
\hline $\mathrm{H} 4 \mathrm{~A}$ & 0.2705 & -0.031 & 0.3188 & $0.07^{*}$ \\
\hline H4B & 0.3192 & -0.0763 & 0.2623 & $0.07 *$ \\
\hline $\mathrm{H} 4 \mathrm{C}$ & 0.2233 & -0.0865 & 0.2681 & $0.07^{*}$ \\
\hline N1 & $0.25288(17)$ & $0.00771(16)$ & $0.15858(13)$ & $0.0265(5)$ \\
\hline N2 & $0.24786(16)$ & $0.08720(15)$ & $0.24065(13)$ & $0.0236(5)$ \\
\hline $\mathrm{H} 1 \mathrm{~N}$ & $0.255(2)$ & $-0.0354(12)$ & $0.1362(13)$ & $0.028^{*}$ \\
\hline $\mathrm{H} 2 \mathrm{~N}$ & $0.248(2)$ & $0.1084(15)$ & $0.2801(10)$ & $0.028^{*}$ \\
\hline $\mathrm{C} 5$ & $0.4466(3)$ & $0.0710(3)$ & $0.1457(2)$ & $0.0472(10)$ \\
\hline H5 & 0.4409 & 0.0769 & 0.0984 & $0.057^{*}$ \\
\hline C6 & $0.4432(3)$ & $0.1273(3)$ & $0.1927(2)$ & $0.0429(9)$ \\
\hline H6 & 0.4352 & 0.1801 & 0.1847 & $0.051^{*}$ \\
\hline $\mathrm{C} 7$ & $0.46291(19)$ & $0.0184(2)$ & $0.2452(2)$ & $0.0338(7)$ \\
\hline $\mathrm{C} 8$ & $0.4797(3)$ & $-0.0370(3)$ & $0.3006(2)$ & $0.0458(10)$ \\
\hline $\mathrm{H} 8 \mathrm{~A}$ & 0.5322 & -0.0253 & 0.3215 & $0.069^{*}$ \\
\hline H8B & 0.4807 & -0.0887 & 0.2822 & $0.069^{*}$ \\
\hline $\mathrm{H} 8 \mathrm{C}$ & 0.4372 & -0.0333 & 0.3344 & $0.069^{*}$ \\
\hline N3 & $0.45995(18)$ & 0.00367 (19) & $0.18011(18)$ & $0.0356(7)$ \\
\hline N4 & $0.45352(17)$ & $0.09260(18)$ & $0.25376(18)$ & $0.0343(6)$ \\
\hline $\mathrm{H} 3 \mathrm{~N}$ & $0.457(3)$ & $-0.0409(12)$ & $0.1597(14)$ & $0.041^{*}$ \\
\hline $\mathrm{H} 4 \mathrm{~N}$ & $0.450(3)$ & $0.1166(16)$ & $0.2926(10)$ & $0.041^{*}$ \\
\hline C9 & $0.4628(3)$ & $0.5730(2)$ & $0.3839(2)$ & $0.0417(9)$ \\
\hline H9 & 0.4661 & 0.5816 & 0.431 & $0.05^{*}$ \\
\hline C10 & $0.4672(2)$ & $0.6261(2)$ & $0.3346(2)$ & $0.0389(8)$ \\
\hline H10 & 0.4739 & 0.6794 & 0.3407 & $0.047^{*}$ \\
\hline $\mathrm{C} 11$ & $0.45236(19)$ & $0.5147(2)$ & $0.28609(19)$ & $0.0308(7)$ \\
\hline $\mathrm{C} 12$ & $0.4469(3)$ & $0.4532(3)$ & $0.2355(2)$ & $0.0536(12)$ \\
\hline $\mathrm{H} 12 \mathrm{~A}$ & 0.4124 & 0.4697 & 0.1979 & $0.08^{*}$ \\
\hline H12B & 0.4235 & 0.4077 & 0.2561 & $0.08 *$ \\
\hline $\mathrm{H} 12 \mathrm{C}$ & 0.5011 & 0.4413 & 0.219 & $0.08 *$ \\
\hline N5 & $0.45244(17)$ & $0.50405(18)$ & $0.35196(16)$ & $0.0316(6)$ \\
\hline N6 & $0.46028(17)$ & $0.58857(17)$ & $0.27414(16)$ & $0.0316(6)$ \\
\hline $\mathrm{H} 5 \mathrm{~N}$ & $0.446(3)$ & $0.4604(12)$ & $0.3735(13)$ & $0.038^{*}$ \\
\hline $\mathrm{H} 6 \mathrm{~N}$ & $0.456(3)$ & $0.6126(15)$ & $0.2346(10)$ & $0.038^{*}$ \\
\hline $\mathrm{C} 13$ & $0.4092(3)$ & $0.3119(4)$ & $0.0588(3)$ & $0.0606(14)$ \\
\hline H13 & 0.4644 & 0.327 & 0.0599 & $0.073^{*}$ \\
\hline $\mathrm{C} 14$ & $0.3540(3)$ & $0.3234(3)$ & $0.1070(3)$ & $0.0549(12)$ \\
\hline H14 & 0.363 & 0.3476 & 0.1492 & $0.066^{*}$ \\
\hline $\mathrm{C} 15$ & $0.2921(3)$ & $0.2642(2)$ & $0.02298(19)$ & $0.0364(8)$ \\
\hline $\mathrm{C} 16$ & $0.2296(4)$ & $0.2267(3)$ & $-0.0190(3)$ & $0.0564(12)$ \\
\hline $\mathrm{H} 16 \mathrm{~A}$ & 0.2271 & 0.1726 & -0.0075 & $0.085^{*}$ \\
\hline
\end{tabular}




\begin{tabular}{|c|c|c|c|c|}
\hline H16B & 0.2433 & 0.2323 & -0.0666 & $0.085^{*}$ \\
\hline $\mathrm{H} 16 \mathrm{C}$ & 0.177 & 0.2503 & -0.0108 & $0.085^{*}$ \\
\hline N7 & $0.3698(2)$ & $0.2740(2)$ & 0.00784 (19) & $0.0475(9)$ \\
\hline N8 & $0.2818(2)$ & $0.2935(2)$ & $0.08359(17)$ & $0.0398(7)$ \\
\hline $\mathrm{H} 7 \mathrm{~N}$ & $0.3920(18)$ & $0.263(3)$ & $-0.0313(13)$ & $0.048^{*}$ \\
\hline $\mathrm{H} 8 \mathrm{~N}$ & $0.2341(13)$ & $0.301(3)$ & $0.1029(17)$ & $0.048^{*}$ \\
\hline $\mathrm{C} 17$ & $0.3403(2)$ & $0.3084(2)$ & $0.75070(18)$ & $0.0319(7)$ \\
\hline H17 & 0.3787 & 0.2916 & 0.7834 & $0.038^{*}$ \\
\hline $\mathrm{C} 18$ & $0.34305(19)$ & $0.2954(2)$ & $0.68388(18)$ & $0.0300(6)$ \\
\hline H18 & 0.3836 & 0.2681 & 0.6606 & $0.036^{*}$ \\
\hline C19 & $0.23323(18)$ & $0.36274(19)$ & $0.70469(15)$ & $0.0251(5)$ \\
\hline $\mathrm{C} 20$ & $0.1568(2)$ & $0.4053(3)$ & $0.6948(2)$ & $0.0404(9)$ \\
\hline $\mathrm{H} 20 \mathrm{~A}$ & 0.119 & 0.392 & 0.7305 & $0.061^{*}$ \\
\hline $\mathrm{H} 20 \mathrm{~B}$ & 0.1328 & 0.392 & 0.651 & $0.061^{*}$ \\
\hline $\mathrm{H} 20 \mathrm{C}$ & 0.1679 & 0.46 & 0.6962 & $0.061 *$ \\
\hline N9 & $0.27156(17)$ & $0.35055(18)$ & $0.76280(14)$ & $0.0292(5)$ \\
\hline N10 & $0.27544(16)$ & $0.32947(17)$ & $0.65606(13)$ & $0.0260(5)$ \\
\hline $\mathrm{H} 9 \mathrm{~N}$ & $0.2597(19)$ & $0.369(2)$ & $0.8029(10)$ & $0.031^{*}$ \\
\hline $\mathrm{H} 10 \mathrm{~N}$ & $0.2612(19)$ & $0.328(2)$ & $0.6133(9)$ & $0.031^{*}$ \\
\hline $\mathrm{C} 21$ & $0.1295(2)$ & $0.2036(2)$ & $0.62572(16)$ & $0.0294(6)$ \\
\hline $\mathrm{H} 21$ & 0.0909 & 0.2298 & 0.5985 & $0.035^{*}$ \\
\hline $\mathrm{C} 22$ & 0.12795 (19) & $0.1954(2)$ & $0.69351(15)$ & $0.0289(6)$ \\
\hline $\mathrm{H} 22$ & 0.0878 & 0.2144 & 0.7228 & $0.035^{*}$ \\
\hline $\mathrm{C} 23$ & 0.23697 (19) & $0.13713(18)$ & $0.65689(14)$ & $0.0241(5)$ \\
\hline $\mathrm{C} 24$ & $0.3139(2)$ & $0.0943(2)$ & $0.6550(2)$ & $0.0356(7)$ \\
\hline $\mathrm{H} 24 \mathrm{~A}$ & 0.3575 & 0.1257 & 0.6739 & $0.053^{*}$ \\
\hline H24B & 0.3264 & 0.0813 & 0.6083 & $0.053^{*}$ \\
\hline $\mathrm{H} 24 \mathrm{C}$ & 0.3088 & 0.0476 & 0.6815 & $0.053^{*}$ \\
\hline N11 & $0.19766(16)$ & $0.16674(17)$ & $0.60401(12)$ & $0.0262(5)$ \\
\hline N12 & $0.19571(16)$ & $0.15416(17)$ & $0.71190(12)$ & $0.0257(5)$ \\
\hline $\mathrm{H} 11 \mathrm{~N}$ & $0.2134(19)$ & $0.164(2)$ & $0.5621(9)$ & $0.031^{*}$ \\
\hline $\mathrm{H} 12 \mathrm{~N}$ & $0.2122(19)$ & $0.145(2)$ & $0.7531(9)$ & $0.031^{*}$ \\
\hline $\mathrm{C} 25$ & $0.3331(3)$ & $-0.0694(3)$ & $0.5204(2)$ & $0.0543(12)$ \\
\hline H25 & 0.3753 & -0.0638 & 0.4888 & $0.065^{*}$ \\
\hline $\mathrm{C} 26$ & $0.3380(3)$ & $-0.1023(3)$ & $0.5821(2)$ & $0.0498(11)$ \\
\hline H26 & 0.3853 & -0.1248 & 0.6009 & $0.06^{*}$ \\
\hline $\mathrm{C} 27$ & $0.2140(3)$ & $-0.0632(2)$ & $0.5699(2)$ & $0.0439(10)$ \\
\hline $\mathrm{C} 28$ & $0.1286(4)$ & $-0.0448(3)$ & $0.5815(3)$ & $0.0625(14)$ \\
\hline $\mathrm{H} 28 \mathrm{~A}$ & 0.0994 & -0.0912 & 0.5938 & $0.094 *$ \\
\hline $\mathrm{H} 28 \mathrm{~B}$ & 0.1046 & -0.0234 & 0.5404 & $0.094^{*}$ \\
\hline $\mathrm{H} 28 \mathrm{C}$ & 0.1248 & -0.0077 & 0.618 & $0.094 *$ \\
\hline N13 & $0.2543(3)$ & $-0.0461(2)$ & $0.51329(18)$ & $0.0523(10)$ \\
\hline N14 & $0.2641(2)$ & $-0.0981(2)$ & $0.61320(16)$ & $0.0421(8)$ \\
\hline $\mathrm{H} 13 \mathrm{~N}$ & $0.2347(18)$ & $-0.022(3)$ & $0.4779(15)$ & $0.05^{*}$ \\
\hline $\mathrm{C} 29$ & $0.1459(2)$ & $0.5736(2)$ & $0.59154(18)$ & $0.0331(7)$ \\
\hline H29 & 0.0966 & 0.5697 & 0.5669 & $0.04 *$ \\
\hline C30 & $0.1591(2)$ & $0.6127(2)$ & $0.64964(18)$ & $0.0325(7)$ \\
\hline H30 & 0.1195 & 0.6411 & 0.673 & $0.039^{*}$ \\
\hline
\end{tabular}




\begin{tabular}{lllll}
$\mathrm{C} 31$ & $0.2732(2)$ & $0.5611(2)$ & $0.62385(16)$ & $0.0289(6)$ \\
$\mathrm{C} 32$ & $0.3600(2)$ & $0.5372(3)$ & $0.6246(2)$ & $0.0472(11)$ \\
$\mathrm{H} 32 \mathrm{~A}$ & 0.3916 & 0.5723 & 0.6526 & $0.071^{*}$ \\
$\mathrm{H} 32 \mathrm{~B}$ & 0.3807 & 0.5378 & 0.5786 & $0.071^{*}$ \\
$\mathrm{H} 32 \mathrm{C}$ & 0.3645 & 0.4856 & 0.643 & $0.071^{*}$ \\
$\mathrm{~N} 15$ & $0.21866(17)$ & $0.54082(17)$ & $0.57597(14)$ & $0.0287(5)$ \\
$\mathrm{N} 16$ & $0.23894(17)$ & $0.60467(18)$ & $0.66955(14)$ & $0.0302(6)$ \\
$\mathrm{H} 15 \mathrm{~N}$ & $0.2266(18)$ & $0.511(2)$ & $0.5419(15)$ & $0.036^{*}$ \\
$\mathrm{O} 25$ & $0.14267(16)$ & $0.30433(19)$ & $0.14871(12)$ & $0.0369(6)$ \\
$\mathrm{H} 25 \mathrm{~V}$ & $0.134(3)$ & $0.300(3)$ & $0.1909(10)$ & $0.055^{*}$ \\
$\mathrm{H} 25 \mathrm{~W}$ & $0.0957(17)$ & $0.302(3)$ & $0.130(2)$ & $0.055^{*}$ \\
$\mathrm{O} 26$ & $0.4963(2)$ & $0.2100(2)$ & $0.58818(17)$ & $0.0519(8)$ \\
$\mathrm{H} 26 \mathrm{~V}$ & $0.482(4)$ & $0.248(2)$ & $0.566(3)$ & $0.078^{*}$ \\
$\mathrm{H} 26 \mathrm{~W}$ & $0.482(4)$ & $0.172(2)$ & $0.566(3)$ & $0.078^{*}$ \\
\hline
\end{tabular}

Atomic displacement parameters $\left(\AA^{2}\right)$

\begin{tabular}{|c|c|c|c|c|c|c|}
\hline & $U^{11}$ & $U^{22}$ & $U^{33}$ & $U^{12}$ & $U^{13}$ & $U^{23}$ \\
\hline $\mathrm{O} 1$ & $0.0214(9)$ & $0.0333(12)$ & $0.0294(11)$ & $-0.0021(8)$ & $0.0030(8)$ & $0.0072(9)$ \\
\hline $\mathrm{O} 2$ & $0.0330(11)$ & $0.0339(12)$ & $0.0182(9)$ & $-0.0065(9)$ & $-0.0104(8)$ & $0.0047(8)$ \\
\hline $\mathrm{O} 3$ & $0.0288(10)$ & $0.0217(9)$ & $0.0183(9)$ & $-0.0051(8)$ & $-0.0051(7)$ & $0.0004(7)$ \\
\hline $\mathrm{O} 4$ & $0.0258(10)$ & $0.0345(12)$ & $0.0206(9)$ & $-0.0030(9)$ & $0.0017(8)$ & $0.0070(8)$ \\
\hline O5 & $0.0382(12)$ & $0.0218(10)$ & $0.0285(11)$ & $-0.0060(9)$ & $-0.0035(9)$ & $-0.0020(8)$ \\
\hline O6 & $0.0218(8)$ & $0.0208(9)$ & $0.0129(7)$ & $-0.0003(7)$ & $0.0016(6)$ & $0.0024(6)$ \\
\hline $\mathrm{O} 7$ & $0.0255(10)$ & $0.0328(12)$ & $0.0268(11)$ & $0.0018(9)$ & $-0.0034(8)$ & $0.0067(9)$ \\
\hline O8 & $0.0375(12)$ & $0.0221(10)$ & $0.0247(10)$ & $0.0050(9)$ & $0.0080(9)$ & $0.0008(8)$ \\
\hline O9 & $0.0295(10)$ & $0.0258(10)$ & $0.0253(10)$ & $0.0044(8)$ & $0.0119(8)$ & $0.0025(8)$ \\
\hline $\mathrm{O} 10$ & $0.0240(11)$ & $0.0398(14)$ & $0.0452(15)$ & $0.0009(10)$ & $-0.0018(10)$ & $0.0059(12)$ \\
\hline O11 & $0.0371(13)$ & $0.0433(15)$ & $0.0379(14)$ & $0.0011(11)$ & $0.0227(11)$ & $0.0077(11)$ \\
\hline $\mathrm{O} 12$ & $0.0239(9)$ & $0.0206(9)$ & $0.0223(9)$ & $-0.0029(7)$ & $0.0041(7)$ & $0.0025(7)$ \\
\hline $\mathrm{O} 13$ & $0.0233(10)$ & $0.0389(13)$ & $0.0206(9)$ & $-0.0016(9)$ & $-0.0060(8)$ & $0.0031(9)$ \\
\hline O14 & $0.0312(10)$ & $0.0204(9)$ & $0.0222(9)$ & $-0.0040(8)$ & $-0.0020(8)$ & $-0.0034(7)$ \\
\hline O15 & $0.0219(9)$ & $0.0257(9)$ & $0.0116(7)$ & $-0.0001(7)$ & $-0.0011(6)$ & $0.0004(7)$ \\
\hline O16 & $0.0256(10)$ & $0.0402(13)$ & $0.0177(9)$ & $0.0037(9)$ & $0.0059(8)$ & $0.0036(8)$ \\
\hline $\mathrm{O} 17$ & $0.0310(11)$ & $0.0228(10)$ & $0.0215(9)$ & $0.0033(8)$ & $-0.0038(8)$ & $-0.0032(7)$ \\
\hline $\mathrm{O} 18$ & $0.0242(9)$ & $0.0206(9)$ & $0.0189(9)$ & $0.0013(7)$ & $-0.0031(7)$ & 0.0008 (7) \\
\hline O19 & $0.0306(10)$ & $0.0237(9)$ & $0.0137(8)$ & $-0.0029(8)$ & $-0.0038(7)$ & $0.0018(7)$ \\
\hline $\mathrm{O} 20$ & $0.0283(9)$ & $0.0160(8)$ & $0.0129(7)$ & $-0.0013(7)$ & $0.0032(7)$ & $-0.0007(6)$ \\
\hline $\mathrm{O} 21$ & $0.0321(11)$ & $0.0255(10)$ & $0.0186(9)$ & $0.0004(8)$ & $0.0110(8)$ & $0.0020(7)$ \\
\hline $\mathrm{O} 22$ & $0.0229(8)$ & $0.0167(8)$ & $0.0117(7)$ & $-0.0007(6)$ & $0.0003(6)$ & $-0.0007(6)$ \\
\hline $\mathrm{O} 23$ & $0.0187(8)$ & $0.0189(8)$ & $0.0117(7)$ & $-0.0014(6)$ & $0.0007(6)$ & $0.0008(6)$ \\
\hline $\mathrm{O} 24$ & $0.0198(8)$ & $0.0175(8)$ & $0.0143(7)$ & $0.0004(6)$ & $0.0029(6)$ & $0.0014(6)$ \\
\hline Mo1 & $0.01912(9)$ & $0.02215(10)$ & $0.01584(9)$ & $-0.00268(8)$ & $-0.00351(7)$ & $0.00174(7)$ \\
\hline Mo2 & $0.02159(10)$ & $0.01762(9)$ & $0.01494(9)$ & $-0.00348(7)$ & $-0.00019(7)$ & $0.00209(7)$ \\
\hline Mo3 & $0.02075(10)$ & $0.01832(10)$ & $0.01763(9)$ & $0.00146(8)$ & $0.00352(7)$ & $0.00293(7)$ \\
\hline Mo4 & $0.01970(10)$ & $0.02472(11)$ & $0.02561(11)$ & $0.00006(8)$ & $0.00868(8)$ & $0.00360(9)$ \\
\hline Mo5 & $0.01950(9)$ & $0.01916(10)$ & $0.01367(9)$ & $-0.00115(7)$ & $-0.00133(7)$ & $-0.00076(7)$ \\
\hline Mo6 & $0.01876(9)$ & $0.01945(10)$ & $0.01188(8)$ & $0.00120(7)$ & $0.00054(7)$ & $-0.00103(7)$ \\
\hline
\end{tabular}




\begin{tabular}{|c|c|c|c|c|c|c|}
\hline Mo7 & $0.02116(9)$ & $0.01513(9)$ & $0.01035(8)$ & $-0.00110(7)$ & $0.00168(7)$ & $-0.00035(6)$ \\
\hline $\mathrm{C} 1$ & $0.069(3)$ & $0.0360(19)$ & $0.0254(16)$ & $0.0063(19)$ & $-0.0037(17)$ & $-0.0048(14)$ \\
\hline $\mathrm{C} 2$ & $0.054(2)$ & $0.0268(15)$ & $0.0291(16)$ & $0.0069(15)$ & $-0.0016(15)$ & $-0.0060(12)$ \\
\hline $\mathrm{C} 3$ & $0.0225(12)$ & $0.0242(13)$ & $0.0263(13)$ & $0.0004(10)$ & $0.0014(10)$ & $-0.0075(10)$ \\
\hline $\mathrm{C} 4$ & $0.076(3)$ & $0.0280(17)$ & $0.036(2)$ & $-0.0038(18)$ & $0.002(2)$ & $-0.0050(15)$ \\
\hline N1 & $0.0293(12)$ & $0.0281(12)$ & $0.0222(11)$ & $0.0017(10)$ & $0.0000(9)$ & $-0.0128(9)$ \\
\hline N2 & $0.0248(11)$ & $0.0248(11)$ & $0.0211(11)$ & $0.0002(9)$ & $0.0014(9)$ & $-0.0092(9)$ \\
\hline $\mathrm{C} 5$ & $0.052(2)$ & $0.051(3)$ & $0.038(2)$ & $0.000(2)$ & $-0.0123(18)$ & $-0.0113(18)$ \\
\hline C6 & $0.047(2)$ & $0.041(2)$ & $0.041(2)$ & $0.0030(17)$ & $-0.0089(17)$ & $-0.0061(17)$ \\
\hline $\mathrm{C} 7$ & $0.0215(13)$ & $0.0335(17)$ & $0.047(2)$ & $-0.0041(12)$ & $0.0096(13)$ & $-0.0113(14)$ \\
\hline $\mathrm{C} 8$ & $0.045(2)$ & $0.046(2)$ & $0.046(2)$ & $0.0079(18)$ & $0.0100(18)$ & $-0.0030(18)$ \\
\hline N3 & $0.0256(13)$ & $0.0373(16)$ & $0.0439(17)$ & $-0.0015(11)$ & $0.0025(12)$ & $-0.0179(13)$ \\
\hline N4 & $0.0231(12)$ & $0.0330(15)$ & $0.0469(18)$ & $-0.0027(11)$ & $0.0027(11)$ & $-0.0127(13)$ \\
\hline C9 & $0.050(2)$ & $0.037(2)$ & $0.038(2)$ & $-0.0067(17)$ & $0.0036(17)$ & $0.0054(15)$ \\
\hline $\mathrm{C} 10$ & $0.043(2)$ & $0.0313(17)$ & $0.043(2)$ & $-0.0031(15)$ & $0.0023(16)$ & $0.0063(15)$ \\
\hline C11 & $0.0230(13)$ & $0.0311(15)$ & $0.0380(17)$ & $-0.0034(11)$ & $-0.0103(12)$ & $0.0118(13)$ \\
\hline $\mathrm{C} 12$ & $0.073(3)$ & $0.040(2)$ & $0.047(2)$ & $-0.004(2)$ & $-0.020(2)$ & $0.0017(19)$ \\
\hline N5 & $0.0250(12)$ & $0.0308(14)$ & $0.0389(16)$ & $-0.0056(10)$ & $-0.0032(11)$ & $0.0133(12)$ \\
\hline N6 & $0.0261(12)$ & $0.0306(14)$ & $0.0380(15)$ & $-0.0036(10)$ & $-0.0054(11)$ & $0.0120(12)$ \\
\hline $\mathrm{C} 13$ & $0.037(2)$ & $0.077(4)$ & $0.068(3)$ & $0.002(2)$ & $0.012(2)$ & $-0.008(3)$ \\
\hline $\mathrm{C} 14$ & $0.045(2)$ & $0.070(3)$ & $0.050(3)$ & $-0.003(2)$ & $0.005(2)$ & $-0.020(2)$ \\
\hline $\mathrm{C} 15$ & $0.047(2)$ & $0.0326(17)$ & $0.0296(16)$ & $0.0093(15)$ & $0.0081(14)$ & $0.0016(13)$ \\
\hline $\mathrm{C} 16$ & $0.077(4)$ & $0.044(3)$ & $0.047(3)$ & $0.002(2)$ & $-0.011(2)$ & $-0.005(2)$ \\
\hline N7 & $0.053(2)$ & $0.052(2)$ & $0.0381(18)$ & $0.0154(17)$ & $0.0202(16)$ & $0.0027(16)$ \\
\hline N8 & $0.0393(16)$ & 0.0454 (19) & $0.0350(16)$ & $0.0044(14)$ & $0.0116(13)$ & $-0.0041(14)$ \\
\hline $\mathrm{C} 17$ & $0.0242(13)$ & $0.0393(18)$ & $0.0321(16)$ & $0.0027(12)$ & $-0.0053(12)$ & $0.0007(13)$ \\
\hline $\mathrm{C} 18$ & $0.0220(13)$ & $0.0359(17)$ & $0.0322(16)$ & $0.0035(12)$ & $0.0030(11)$ & $-0.0006(13)$ \\
\hline C19 & $0.0228(12)$ & $0.0309(15)$ & $0.0215(12)$ & $0.0022(11)$ & $0.0018(10)$ & $-0.0001(10)$ \\
\hline $\mathrm{C} 20$ & $0.0333(17)$ & $0.048(2)$ & $0.040(2)$ & $0.0146(16)$ & $-0.0022(14)$ & $0.0057(17)$ \\
\hline N9 & $0.0293(13)$ & $0.0380(15)$ & $0.0203(11)$ & $0.0040(11)$ & $-0.0012(9)$ & $-0.0007(10)$ \\
\hline N10 & $0.0266(12)$ & $0.0338(13)$ & $0.0177(10)$ & $-0.0006(10)$ & $0.0024(9)$ & $-0.0001(9)$ \\
\hline $\mathrm{C} 21$ & $0.0277(14)$ & $0.0417(18)$ & $0.0190(12)$ & $0.0009(13)$ & $0.0000(10)$ & $0.0026(12)$ \\
\hline $\mathrm{C} 22$ & $0.0268(13)$ & $0.0415(18)$ & $0.0187(12)$ & $-0.0004(12)$ & $0.0069(10)$ & $0.0002(11)$ \\
\hline $\mathrm{C} 23$ & $0.0288(13)$ & $0.0282(14)$ & $0.0155(11)$ & $-0.0015(11)$ & $0.0017(10)$ & $-0.0007(9)$ \\
\hline $\mathrm{C} 24$ & $0.0362(17)$ & $0.0365(18)$ & $0.0343(17)$ & $0.0089(14)$ & $0.0056(14)$ & $0.0012(14)$ \\
\hline N11 & $0.0289(12)$ & $0.0374(14)$ & $0.0124(9)$ & $-0.0002(10)$ & $0.0027(8)$ & $0.0008(9)$ \\
\hline N12 & $0.0293(12)$ & $0.0347(14)$ & $0.0132(9)$ & $-0.0017(10)$ & $0.0013(8)$ & $0.0037(9)$ \\
\hline $\mathrm{C} 25$ & $0.081(3)$ & $0.039(2)$ & $0.043(2)$ & $0.008(2)$ & $0.026(2)$ & $0.0121(18)$ \\
\hline C26 & $0.066(3)$ & $0.042(2)$ & $0.042(2)$ & $0.003(2)$ & $0.013(2)$ & $0.0089(18)$ \\
\hline $\mathrm{C} 27$ & $0.074(3)$ & $0.0266(16)$ & $0.0306(17)$ & $-0.0031(18)$ & $0.0014(18)$ & $0.0015(13)$ \\
\hline $\mathrm{C} 28$ & $0.075(4)$ & $0.054(3)$ & $0.058(3)$ & $-0.001(3)$ & $-0.006(3)$ & $0.005(2)$ \\
\hline N13 & $0.095(3)$ & $0.0330(17)$ & $0.0288(16)$ & $0.0064(19)$ & $0.0075(18)$ & $0.0112(13)$ \\
\hline N14 & $0.065(2)$ & $0.0348(16)$ & $0.0260(14)$ & $-0.0038(15)$ & $0.0053(14)$ & $0.0052(12)$ \\
\hline $\mathrm{C} 29$ & $0.0285(15)$ & $0.0419(19)$ & $0.0286(15)$ & $0.0052(13)$ & $-0.0094(12)$ & $-0.0013(13)$ \\
\hline C30 & $0.0307(15)$ & $0.0369(17)$ & $0.0298(15)$ & $0.0115(13)$ & $-0.0028(12)$ & $-0.0057(13)$ \\
\hline C31 & $0.0290(14)$ & $0.0355(16)$ & $0.0222(13)$ & $0.0043(12)$ & $-0.0059(11)$ & $-0.0094(12)$ \\
\hline $\mathrm{C} 32$ & $0.0322(18)$ & $0.068(3)$ & $0.041(2)$ & $0.0155(19)$ & $-0.0086(15)$ & $-0.019(2)$ \\
\hline N15 & $0.0315(13)$ & $0.0327(14)$ & $0.0217(11)$ & $0.0048(11)$ & $-0.0046(10)$ & $-0.0109(10)$ \\
\hline
\end{tabular}




\begin{tabular}{lllllll}
$\mathrm{N} 16$ & $0.0302(13)$ & $0.0364(15)$ & $0.0240(12)$ & $0.0088(11)$ & $-0.0057(10)$ & $-0.0092(11)$ \\
$\mathrm{O} 25$ & $0.0344(12)$ & $0.0563(17)$ & $0.0199(10)$ & $0.0001(12)$ & $0.0025(9)$ & $0.0003(11)$ \\
$\mathrm{O} 26$ & $0.066(2)$ & $0.0466(18)$ & $0.0422(17)$ & $-0.0038(16)$ & $-0.0272(15)$ & $0.0040(14)$ \\
\hline
\end{tabular}

Geometric parameters $\left(A,{ }^{o}\right)$

\begin{tabular}{|c|c|c|c|}
\hline $\mathrm{O} 1-\mathrm{Mo} 1$ & $1.724(2)$ & $\mathrm{C} 11-\mathrm{N} 6$ & $1.328(4)$ \\
\hline $\mathrm{O} 2-\mathrm{Mo} 1$ & $1.725(2)$ & $\mathrm{C} 11-\mathrm{C} 12$ & $1.479(6)$ \\
\hline $\mathrm{O} 3-\mathrm{Mo} 1$ & $1.945(2)$ & $\mathrm{C} 12-\mathrm{H} 12 \mathrm{~A}$ & 0.98 \\
\hline $\mathrm{O} 3-\mathrm{Mo} 2$ & $1.971(2)$ & $\mathrm{C} 12-\mathrm{H} 12 \mathrm{~B}$ & 0.98 \\
\hline $\mathrm{O} 4-\mathrm{Mo} 2$ & $1.707(2)$ & $\mathrm{C} 12-\mathrm{H} 12 \mathrm{C}$ & 0.98 \\
\hline $\mathrm{O} 5-\mathrm{Mo} 2$ & $1.725(2)$ & $\mathrm{N} 5-\mathrm{H} 5 \mathrm{~N}$ & $0.886(17)$ \\
\hline O6-Mo3 & $1.9528(19)$ & $\mathrm{N} 6-\mathrm{H} 6 \mathrm{~N}$ & $0.895(17)$ \\
\hline $\mathrm{O} 6-\mathrm{Mo} 2$ & $1.953(2)$ & $\mathrm{C} 13-\mathrm{C} 14$ & $1.347(7)$ \\
\hline $\mathrm{O} 7-\mathrm{Mo} 3$ & $1.715(2)$ & $\mathrm{C} 13-\mathrm{N} 7$ & $1.372(7)$ \\
\hline $\mathrm{O} 8-\mathrm{Mo} 3$ & $1.716(2)$ & $\mathrm{C} 13-\mathrm{H} 13$ & 0.95 \\
\hline O9-Mo4 & $1.939(2)$ & $\mathrm{C} 14-\mathrm{N} 8$ & $1.379(6)$ \\
\hline O9-Mo3 & $1.987(2)$ & $\mathrm{C} 14-\mathrm{H} 14$ & 0.95 \\
\hline O10-Mo4 & $1.709(3)$ & $\mathrm{C} 15-\mathrm{N} 8$ & $1.324(5)$ \\
\hline $\mathrm{O} 11-\mathrm{Mo} 4$ & $1.715(2)$ & $\mathrm{C} 15-\mathrm{N} 7$ & $1.333(5)$ \\
\hline O12-Mo4 & $1.932(2)$ & $\mathrm{C} 15-\mathrm{C} 16$ & $1.476(6)$ \\
\hline O12-Mo5 & $1.983(2)$ & $\mathrm{C} 16-\mathrm{H} 16 \mathrm{~A}$ & 0.98 \\
\hline O13-Mo5 & $1.713(2)$ & $\mathrm{C} 16-\mathrm{H} 16 \mathrm{~B}$ & 0.98 \\
\hline O14-Mo5 & $1.730(2)$ & $\mathrm{C} 16-\mathrm{H} 16 \mathrm{C}$ & 0.98 \\
\hline O15-Mo6 & $1.9521(19)$ & $\mathrm{N} 7-\mathrm{H} 7 \mathrm{~N}$ & $0.887(17)$ \\
\hline O15-Mo5 & $1.953(2)$ & $\mathrm{N} 8-\mathrm{H} 8 \mathrm{~N}$ & $0.893(17)$ \\
\hline O16-Mo6 & $1.707(2)$ & $\mathrm{C} 17-\mathrm{C} 18$ & $1.350(5)$ \\
\hline O17-Mo6 & $1.726(2)$ & $\mathrm{C} 17-\mathrm{N} 9$ & $1.380(4)$ \\
\hline O18-Mo1 & $1.916(2)$ & $\mathrm{C} 17-\mathrm{H} 17$ & 0.95 \\
\hline O18-Mo6 & $2.012(2)$ & $\mathrm{C} 18-\mathrm{N} 10$ & $1.379(4)$ \\
\hline $\mathrm{O} 19-\mathrm{Mo} 7$ & $1.754(2)$ & C18-H18 & 0.95 \\
\hline O19-Mo1 & $2.453(2)$ & $\mathrm{C} 19-\mathrm{N} 9$ & $1.329(4)$ \\
\hline $\mathrm{O} 20-\mathrm{Mo} 7$ & $1.901(2)$ & $\mathrm{C} 19-\mathrm{N} 10$ & $1.334(4)$ \\
\hline $\mathrm{O} 20-\mathrm{Mo} 3$ & $2.259(2)$ & $\mathrm{C} 19-\mathrm{C} 20$ & $1.480(5)$ \\
\hline $\mathrm{O} 20-\mathrm{Mo} 2$ & $2.292(2)$ & $\mathrm{C} 20-\mathrm{H} 20 \mathrm{~A}$ & 0.98 \\
\hline $\mathrm{O} 21-\mathrm{Mo} 7$ & $1.725(2)$ & $\mathrm{C} 20-\mathrm{H} 20 \mathrm{~B}$ & 0.98 \\
\hline $\mathrm{O} 22-\mathrm{Mo} 7$ & $1.8945(19)$ & $\mathrm{C} 20-\mathrm{H} 20 \mathrm{C}$ & 0.98 \\
\hline $\mathrm{O} 22-\mathrm{Mo} 5$ & $2.2453(19)$ & N9- $\mathrm{H} 9 \mathrm{~N}$ & $0.886(17)$ \\
\hline O22-Mo6 & $2.3057(19)$ & $\mathrm{N} 10-\mathrm{H} 10 \mathrm{~N}$ & $0.880(17)$ \\
\hline O23-Mo6 & $2.1329(19)$ & $\mathrm{C} 21-\mathrm{C} 22$ & $1.356(4)$ \\
\hline $\mathrm{O} 23-\mathrm{Mo} 1$ & $2.1604(19)$ & $\mathrm{C} 21-\mathrm{N} 11$ & $1.374(4)$ \\
\hline $\mathrm{O} 23-\mathrm{Mo} 2$ & $2.1748(19)$ & $\mathrm{C} 21-\mathrm{H} 21$ & 0.95 \\
\hline $\mathrm{O} 23-\mathrm{Mo} 7$ & $2.3011(18)$ & $\mathrm{C} 22-\mathrm{N} 12$ & $1.380(4)$ \\
\hline $\mathrm{O} 24-\mathrm{Mo} 4$ & $2.1624(19)$ & $\mathrm{C} 22-\mathrm{H} 22$ & 0.95 \\
\hline $\mathrm{O} 24-\mathrm{Mo} 5$ & $2.1665(19)$ & $\mathrm{C} 23-\mathrm{N} 12$ & $1.330(4)$ \\
\hline $\mathrm{O} 24-\mathrm{Mo3}$ & $2.1677(19)$ & $\mathrm{C} 23-\mathrm{N} 11$ & $1.336(4)$ \\
\hline $\mathrm{O} 24-\mathrm{Mo} 7$ & $2.2665(19)$ & $\mathrm{C} 23-\mathrm{C} 24$ & $1.479(5)$ \\
\hline Mo1-Mo6 & $3.2178(3)$ & $\mathrm{C} 24-\mathrm{H} 24 \mathrm{~A}$ & 0.98 \\
\hline
\end{tabular}




\begin{tabular}{|c|c|c|c|}
\hline Mo4-Mo5 & $3.2105(3)$ & $\mathrm{C} 24-\mathrm{H} 24 \mathrm{~B}$ & 0.98 \\
\hline $\mathrm{C} 1-\mathrm{C} 2$ & $1.362(5)$ & $\mathrm{C} 24-\mathrm{H} 24 \mathrm{C}$ & 0.98 \\
\hline $\mathrm{C} 1-\mathrm{N} 1$ & $1.362(5)$ & $\mathrm{N} 11-\mathrm{H} 11 \mathrm{~N}$ & $0.877(17)$ \\
\hline $\mathrm{C} 1-\mathrm{H} 1$ & 0.95 & $\mathrm{~N} 12-\mathrm{H} 12 \mathrm{~N}$ & $0.876(17)$ \\
\hline $\mathrm{C} 2-\mathrm{N} 2$ & $1.365(5)$ & $\mathrm{C} 25-\mathrm{C} 26$ & $1.357(6)$ \\
\hline $\mathrm{C} 2-\mathrm{H} 2$ & 0.95 & $\mathrm{C} 25-\mathrm{N} 13$ & $1.371(7)$ \\
\hline $\mathrm{C} 3-\mathrm{N} 1$ & $1.328(4)$ & $\mathrm{C} 25-\mathrm{H} 25$ & 0.95 \\
\hline $\mathrm{C} 3-\mathrm{N} 2$ & $1.332(4)$ & $\mathrm{C} 26-\mathrm{N} 14$ & $1.377(6)$ \\
\hline $\mathrm{C} 3-\mathrm{C} 4$ & $1.488(5)$ & $\mathrm{C} 26-\mathrm{H} 26$ & 0.95 \\
\hline $\mathrm{C} 4-\mathrm{H} 4 \mathrm{~A}$ & 0.98 & $\mathrm{C} 27-\mathrm{N} 14$ & $1.338(6)$ \\
\hline $\mathrm{C} 4-\mathrm{H} 4 \mathrm{~B}$ & 0.98 & $\mathrm{C} 27-\mathrm{N} 13$ & $1.348(6)$ \\
\hline $\mathrm{C} 4-\mathrm{H} 4 \mathrm{C}$ & 0.98 & $\mathrm{C} 27-\mathrm{C} 28$ & $1.470(8)$ \\
\hline $\mathrm{N} 1-\mathrm{H} 1 \mathrm{~N}$ & $0.881(17)$ & $\mathrm{C} 28-\mathrm{H} 28 \mathrm{~A}$ & 0.98 \\
\hline $\mathrm{N} 2-\mathrm{H} 2 \mathrm{~N}$ & $0.868(17)$ & $\mathrm{C} 28-\mathrm{H} 28 \mathrm{~B}$ & 0.98 \\
\hline $\mathrm{C} 5-\mathrm{C} 6$ & $1.363(6)$ & $\mathrm{C} 28-\mathrm{H} 28 \mathrm{C}$ & 0.98 \\
\hline $\mathrm{C} 5-\mathrm{N} 3$ & $1.384(6)$ & $\mathrm{N} 13-\mathrm{H} 13 \mathrm{~N}$ & $0.880(19)$ \\
\hline $\mathrm{C} 5-\mathrm{H} 5$ & 0.95 & $\mathrm{C} 29-\mathrm{C} 30$ & $1.360(5)$ \\
\hline $\mathrm{C} 6-\mathrm{N} 4$ & $1.367(6)$ & $\mathrm{C} 29-\mathrm{N} 15$ & $1.372(4)$ \\
\hline $\mathrm{C} 6-\mathrm{H} 6$ & 0.95 & $\mathrm{C} 29-\mathrm{H} 29$ & 0.95 \\
\hline $\mathrm{C} 7-\mathrm{N} 3$ & $1.321(5)$ & $\mathrm{C} 30-\mathrm{N} 16$ & $1.380(4)$ \\
\hline $\mathrm{C} 7-\mathrm{N} 4$ & $1.325(5)$ & $\mathrm{C} 30-\mathrm{H} 30$ & 0.95 \\
\hline $\mathrm{C} 7-\mathrm{C} 8$ & $1.493(6)$ & $\mathrm{C} 31-\mathrm{N} 16$ & $1.321(4)$ \\
\hline $\mathrm{C} 8-\mathrm{H} 8 \mathrm{~A}$ & 0.98 & $\mathrm{C} 31-\mathrm{N} 15$ & $1.352(4)$ \\
\hline $\mathrm{C} 8-\mathrm{H} 8 \mathrm{~B}$ & 0.98 & $\mathrm{C} 31-\mathrm{C} 32$ & $1.495(5)$ \\
\hline $\mathrm{C} 8-\mathrm{H} 8 \mathrm{C}$ & 0.98 & $\mathrm{C} 32-\mathrm{H} 32 \mathrm{~A}$ & 0.98 \\
\hline $\mathrm{N} 3-\mathrm{H} 3 \mathrm{~N}$ & $0.884(17)$ & $\mathrm{C} 32-\mathrm{H} 32 \mathrm{~B}$ & 0.98 \\
\hline $\mathrm{N} 4-\mathrm{H} 4 \mathrm{~N}$ & $0.884(17)$ & $\mathrm{C} 32-\mathrm{H} 32 \mathrm{C}$ & 0.98 \\
\hline $\mathrm{C} 9-\mathrm{C} 10$ & $1.357(6)$ & $\mathrm{N} 15-\mathrm{H} 15 \mathrm{~N}$ & $0.867(19)$ \\
\hline $\mathrm{C} 9-\mathrm{N} 5$ & $1.378(5)$ & $\mathrm{O} 25-\mathrm{H} 25 \mathrm{~V}$ & 0.857 (19) \\
\hline C9-H9 & 0.95 & $\mathrm{O} 25-\mathrm{H} 25 \mathrm{~W}$ & $0.856(18)$ \\
\hline $\mathrm{C} 10-\mathrm{N} 6$ & $1.375(5)$ & $\mathrm{O} 26-\mathrm{H} 26 \mathrm{~V}$ & $0.842(19)$ \\
\hline $\mathrm{C} 10-\mathrm{H} 10$ & 0.95 & $\mathrm{O} 26-\mathrm{H} 26 \mathrm{~W}$ & $0.843(19)$ \\
\hline $\mathrm{C} 11-\mathrm{N} 5$ & $1.323(5)$ & & \\
\hline Mo1-O3-Mo2 & $111.13(10)$ & $\mathrm{C} 3-\mathrm{C} 4-\mathrm{H} 4 \mathrm{~A}$ & 109.5 \\
\hline Mo3-O6-Mo2 & $115.96(9)$ & $\mathrm{C} 3-\mathrm{C} 4-\mathrm{H} 4 \mathrm{~B}$ & 109.5 \\
\hline Mo4-O9-Mo3 & $110.89(11)$ & $\mathrm{H} 4 \mathrm{~A}-\mathrm{C} 4-\mathrm{H} 4 \mathrm{~B}$ & 109.5 \\
\hline $\mathrm{Mo} 4-\mathrm{O} 12-\mathrm{Mo5}$ & $110.14(10)$ & $\mathrm{C} 3-\mathrm{C} 4-\mathrm{H} 4 \mathrm{C}$ & 109.5 \\
\hline Mo6-O15-Mo5 & $114.70(9)$ & $\mathrm{H} 4 \mathrm{~A}-\mathrm{C} 4-\mathrm{H} 4 \mathrm{C}$ & 109.5 \\
\hline Mo1-O18-Mo6 & $110.01(10)$ & $\mathrm{H} 4 \mathrm{~B}-\mathrm{C} 4-\mathrm{H} 4 \mathrm{C}$ & 109.5 \\
\hline Mo7-O19-Mo1 & $107.64(9)$ & $\mathrm{C} 3-\mathrm{N} 1-\mathrm{C} 1$ & $109.4(3)$ \\
\hline $\mathrm{Mo} 7-\mathrm{O} 20-\mathrm{Mo} 3$ & $110.29(9)$ & $\mathrm{C} 3-\mathrm{N} 1-\mathrm{H} 1 \mathrm{~N}$ & $125.1(19)$ \\
\hline $\mathrm{Mo} 7-\mathrm{O} 20-\mathrm{Mo} 2$ & $110.20(9)$ & $\mathrm{C} 1-\mathrm{N} 1-\mathrm{H} 1 \mathrm{~N}$ & $125.4(19)$ \\
\hline $\mathrm{Mo} 3-\mathrm{O} 20-\mathrm{Mo} 2$ & $93.36(7)$ & $\mathrm{C} 3-\mathrm{N} 2-\mathrm{C} 2$ & $109.0(3)$ \\
\hline $\mathrm{Mo} 7-\mathrm{O} 22-\mathrm{Mo5}$ & $109.98(9)$ & $\mathrm{C} 3-\mathrm{N} 2-\mathrm{H} 2 \mathrm{~N}$ & $128.7(19)$ \\
\hline Mo7-O22-Mo6 & $109.89(9)$ & $\mathrm{C} 2-\mathrm{N} 2-\mathrm{H} 2 \mathrm{~N}$ & $122.3(19)$ \\
\hline Mo5-O22-Mo6 & $92.51(7)$ & $\mathrm{C} 6-\mathrm{C} 5-\mathrm{N} 3$ & $106.9(4)$ \\
\hline Mo6-O23-Mo1 & $97.09(8)$ & $\mathrm{C} 6-\mathrm{C} 5-\mathrm{H} 5$ & 126.6 \\
\hline
\end{tabular}




$$
\begin{aligned}
& \text { Mo6-O23-Mo2 } \\
& \text { Mo1-O23-Mo2 } \\
& \text { Mo6-O23-Mo7 } \\
& \text { Mo1-O23-Mo7 } \\
& \text { Mo2-O23-Mo7 } \\
& \text { Mo4-O24-Mo5 } \\
& \text { Mo4-O24-Mo3 } \\
& \text { Mo5-O24-Mo3 } \\
& \text { Mo4-O24-Mo7 } \\
& \text { Mo5-O24-Mo7 } \\
& \text { Mo3-O24-Mo7 } \\
& \text { O1-Mo1-O2 } \\
& \text { O1-Mo1-O18 } \\
& \text { O2-Mo1-O18 } \\
& \text { O1-Mo1-O3 } \\
& \text { O2-Mo1-O3 } \\
& \text { O18-Mo1-O3 } \\
& \text { O1-Mo1-O23 } \\
& \text { O2-Mo1-O23 } \\
& \text { O18-Mo1-O23 } \\
& \text { O3-Mo1-O23 } \\
& \text { O1-Mo1-O19 } \\
& \text { O2-Mo1-O19 } \\
& \text { O18-Mo1-O19 } \\
& \text { O3-Mo1-O19 } \\
& \text { O23-Mo1-O19 } \\
& \text { O1-Mo1-Mo6 } \\
& \text { O2-Mo1-Mo6 } \\
& \text { O18-Mo1-Mo6 } \\
& \text { O3-Mo1-Mo6 } \\
& \text { O23-Mo1-Mo6 } \\
& \text { O19-Mo1-Mo6 } \\
& \text { O4-Mo2-O5 } \\
& \text { O4-Mo2-O6 } \\
& \text { O5-Mo2-O6 } \\
& \text { O4-Mo2-O3 } \\
& \text { O5-Mo2-O3 } \\
& \text { O6-Mo2-O3 } \\
& \text { O4-Mo2-O23 } \\
& \text { O5-Mo2-O23 } \\
& \text { O6-Mo2-O23 } \\
& \text { O3-Mo2-O23 } \\
& \text { O4-Mo2-O20 } \\
& \text { O5-Mo2-O20 } \\
& \text { O6-Mo2-O20 } \\
& \text { O3-Mo2-O20 } \\
& \text { O23-Mo2-O20 } \\
& \text { O8-Mo3-O7 }
\end{aligned}
$$

$$
151.14(9)
$$

\begin{tabular}{|c|c|}
\hline $\mathrm{N} 3-\mathrm{C} 5-\mathrm{H} 5$ & 126.6 \\
\hline $\mathrm{C} 5-\mathrm{C} 6-\mathrm{N} 4$ & $106.2(4)$ \\
\hline $\mathrm{C} 5-\mathrm{C} 6-\mathrm{H} 6$ & 126.9 \\
\hline $\mathrm{N} 4-\mathrm{C} 6-\mathrm{H} 6$ & 126.9 \\
\hline $\mathrm{N} 3-\mathrm{C} 7-\mathrm{N} 4$ & $108.4(4)$ \\
\hline $\mathrm{N} 3-\mathrm{C} 7-\mathrm{C} 8$ & $126.8(4)$ \\
\hline $\mathrm{N} 4-\mathrm{C} 7-\mathrm{C} 8$ & $124.7(4)$ \\
\hline $\mathrm{C} 7-\mathrm{C} 8-\mathrm{H} 8 \mathrm{~A}$ & 109.5 \\
\hline $\mathrm{C} 7-\mathrm{C} 8-\mathrm{H} 8 \mathrm{~B}$ & 109.5 \\
\hline $\mathrm{H} 8 \mathrm{~A}-\mathrm{C} 8-\mathrm{H} 8 \mathrm{~B}$ & 109.5 \\
\hline $\mathrm{C} 7-\mathrm{C} 8-\mathrm{H} 8 \mathrm{C}$ & 109.5 \\
\hline $\mathrm{H} 8 \mathrm{~A}-\mathrm{C} 8-\mathrm{H} 8 \mathrm{C}$ & 109.5 \\
\hline $\mathrm{H} 8 \mathrm{~B}-\mathrm{C} 8-\mathrm{H} 8 \mathrm{C}$ & 109.5 \\
\hline $\mathrm{C} 7-\mathrm{N} 3-\mathrm{C} 5$ & $108.7(3)$ \\
\hline $\mathrm{C} 7-\mathrm{N} 3-\mathrm{H} 3 \mathrm{~N}$ & $129(2)$ \\
\hline $\mathrm{C} 5-\mathrm{N} 3-\mathrm{H} 3 \mathrm{~N}$ & $122(2)$ \\
\hline $\mathrm{C} 7-\mathrm{N} 4-\mathrm{C} 6$ & $109.9(3)$ \\
\hline $\mathrm{C} 7-\mathrm{N} 4-\mathrm{H} 4 \mathrm{~N}$ & $126(2)$ \\
\hline $\mathrm{C} 6-\mathrm{N} 4-\mathrm{H} 4 \mathrm{~N}$ & $124(2)$ \\
\hline $\mathrm{C} 10-\mathrm{C} 9-\mathrm{N} 5$ & $106.3(4)$ \\
\hline $\mathrm{C} 10-\mathrm{C} 9-\mathrm{H} 9$ & 126.9 \\
\hline $\mathrm{N} 5-\mathrm{C} 9-\mathrm{H} 9$ & 126.9 \\
\hline $\mathrm{C} 9-\mathrm{C} 10-\mathrm{N} 6$ & $107.2(4)$ \\
\hline $\mathrm{C} 9-\mathrm{C} 10-\mathrm{H} 10$ & 126.4 \\
\hline $\mathrm{N} 6-\mathrm{C} 10-\mathrm{H} 10$ & 126.4 \\
\hline $\mathrm{N} 5-\mathrm{C} 11-\mathrm{N} 6$ & $108.4(3)$ \\
\hline $\mathrm{N} 5-\mathrm{C} 11-\mathrm{C} 12$ & $124.7(3)$ \\
\hline $\mathrm{N} 6-\mathrm{C} 11-\mathrm{C} 12$ & $126.8(4)$ \\
\hline $\mathrm{C} 11-\mathrm{C} 12-\mathrm{H} 12 \mathrm{~A}$ & 109.5 \\
\hline $\mathrm{C} 11-\mathrm{C} 12-\mathrm{H} 12 \mathrm{~B}$ & 109.5 \\
\hline $\mathrm{H} 12 \mathrm{~A}-\mathrm{C} 12-\mathrm{H} 12 \mathrm{~B}$ & 109.5 \\
\hline $\mathrm{C} 11-\mathrm{C} 12-\mathrm{H} 12 \mathrm{C}$ & 109.5 \\
\hline $\mathrm{H} 12 \mathrm{~A}-\mathrm{C} 12-\mathrm{H} 12 \mathrm{C}$ & 109.5 \\
\hline $\mathrm{H} 12 \mathrm{~B}-\mathrm{C} 12-\mathrm{H} 12 \mathrm{C}$ & 109.5 \\
\hline $\mathrm{C} 11-\mathrm{N} 5-\mathrm{C} 9$ & $109.3(3)$ \\
\hline $\mathrm{C} 11-\mathrm{N} 5-\mathrm{H} 5 \mathrm{~N}$ & $127.0(19)$ \\
\hline $\mathrm{C} 9-\mathrm{N} 5-\mathrm{H} 5 \mathrm{~N}$ & $123.7(19)$ \\
\hline $\mathrm{C} 11-\mathrm{N} 6-\mathrm{C} 10$ & $108.7(3)$ \\
\hline $\mathrm{C} 11-\mathrm{N} 6-\mathrm{H} 6 \mathrm{~N}$ & $127.8(19)$ \\
\hline $\mathrm{C} 10-\mathrm{N} 6-\mathrm{H} 6 \mathrm{~N}$ & $123.1(19)$ \\
\hline $\mathrm{C} 14-\mathrm{C} 13-\mathrm{N} 7$ & $106.2(4)$ \\
\hline $\mathrm{C} 14-\mathrm{C} 13-\mathrm{H} 13$ & 126.9 \\
\hline $\mathrm{N} 7-\mathrm{C} 13-\mathrm{H} 13$ & 126.9 \\
\hline $\mathrm{C} 13-\mathrm{C} 14-\mathrm{N} 8$ & $107.0(4)$ \\
\hline $\mathrm{C} 13-\mathrm{C} 14-\mathrm{H} 14$ & 126.5 \\
\hline $\mathrm{N} 8-\mathrm{C} 14-\mathrm{H} 14$ & 126.5 \\
\hline $\mathrm{N} 8-\mathrm{C} 15-\mathrm{N} 7$ & $106.8(4)$ \\
\hline $\mathrm{N} 8-\mathrm{C} 15-\mathrm{C} 16$ & $126.4(4)$ \\
\hline
\end{tabular}$$
96.34(7)
$$$$
101.96(8)
$$$$
100.09 \text { (7) }
$$$$
100.67 \text { (7) }
$$$$
95.74(8)
$$$$
96.60(8)
$$$$
152.34(9)
$$$$
103.86(7)
$$$$
100.00(8)
$$$$
100.92(8)
$$$$
104.12(11)
$$$$
98.92(11)
$$$$
102.57(10)
$$$$
96.55 \text { (11) }
$$$$
100.84(10)
$$$$
147.73(9)
$$$$
106.54(9)
$$$$
149.29(10)
$$$$
74.47(8)
$$$$
74.03(8)
$$$$
176.53(9)
$$$$
78.58(10)
$$$$
82.49(8)
$$$$
80.71(8)
$$$$
70.72(7)
$$$$
95.01(8)
$$$$
137.30(8)
$$$$
35.98(6)
$$$$
114.62(6)
$$$$
41.13(5)
$$$$
84.26(5)
$$$$
105.64(12)
$$$$
96.30(9)
$$$$
97.94 \text { (10) }
$$$$
102.62(10)
$$$$
95.05 \text { (10) }
$$$$
153.21(8)
$$$$
93.82 \text { (10) }
$$$$
159.22(10)
$$$$
86.87 \text { (8) }
$$$$
73.22(8)
$$$$
161.90(10)
$$$$
90.12(10)
$$$$
72.28 \text { (7) }
$$$$
84.40(8)
$$$$
72.01 \text { (7) }
$$$$
104.86 \text { (12) }
$$ 


\begin{tabular}{|c|c|c|c|}
\hline $\mathrm{O} 8-\mathrm{Mo} 3-\mathrm{O} 6$ & $100.55(10)$ & $\mathrm{N} 7-\mathrm{C} 15-\mathrm{C} 16$ & $126.8(4)$ \\
\hline $\mathrm{O} 7-\mathrm{Mo3}-\mathrm{O} 6$ & $96.34(10)$ & $\mathrm{C} 15-\mathrm{C} 16-\mathrm{H} 16 \mathrm{~A}$ & 109.5 \\
\hline $\mathrm{O} 8-\mathrm{Mo} 3-\mathrm{O} 9$ & $92.85(10)$ & $\mathrm{C} 15-\mathrm{C} 16-\mathrm{H} 16 \mathrm{~B}$ & 109.5 \\
\hline $\mathrm{O} 7-\mathrm{Mo3}-\mathrm{O} 9$ & $100.23(11)$ & $\mathrm{H} 16 \mathrm{~A}-\mathrm{C} 16-\mathrm{H} 16 \mathrm{~B}$ & 109.5 \\
\hline $\mathrm{O} 6-\mathrm{Mo} 3-\mathrm{O} 9$ & $155.25(9)$ & $\mathrm{C} 15-\mathrm{C} 16-\mathrm{H} 16 \mathrm{C}$ & 109.5 \\
\hline $\mathrm{O} 8-\mathrm{Mo} 3-\mathrm{O} 24$ & $155.03(9)$ & $\mathrm{H} 16 \mathrm{~A}-\mathrm{C} 16-\mathrm{H} 16 \mathrm{C}$ & 109.5 \\
\hline $\mathrm{O} 7-\mathrm{Mo} 3-\mathrm{O} 24$ & $97.84(10)$ & $\mathrm{H} 16 \mathrm{~B}-\mathrm{C} 16-\mathrm{H} 16 \mathrm{C}$ & 109.5 \\
\hline $\mathrm{O} 6-\mathrm{Mo} 3-\mathrm{O} 24$ & $86.96(8)$ & $\mathrm{C} 15-\mathrm{N} 7-\mathrm{C} 13$ & $110.3(4)$ \\
\hline $\mathrm{O} 9-\mathrm{Mo} 3-\mathrm{O} 24$ & $72.72(8)$ & $\mathrm{C} 15-\mathrm{N} 7-\mathrm{H} 7 \mathrm{~N}$ & $125(2)$ \\
\hline $\mathrm{O} 8-\mathrm{Mo} 3-\mathrm{O} 20$ & $87.48(10)$ & $\mathrm{C} 13-\mathrm{N} 7-\mathrm{H} 7 \mathrm{~N}$ & $124(2)$ \\
\hline $\mathrm{O} 7-\mathrm{Mo} 3-\mathrm{O} 20$ & $165.23(10)$ & $\mathrm{C} 15-\mathrm{N} 8-\mathrm{C} 14$ & $109.7(4)$ \\
\hline $\mathrm{O} 6-\mathrm{Mo} 3-\mathrm{O} 20$ & $73.05(7)$ & $\mathrm{C} 15-\mathrm{N} 8-\mathrm{H} 8 \mathrm{~N}$ & $125(2)$ \\
\hline $\mathrm{O} 9-\mathrm{Mo} 3-\mathrm{O} 20$ & $86.99(9)$ & $\mathrm{C} 14-\mathrm{N} 8-\mathrm{H} 8 \mathrm{~N}$ & $124(2)$ \\
\hline $\mathrm{O} 24-\mathrm{Mo} 3-\mathrm{O} 20$ & $71.84(7)$ & $\mathrm{C} 18-\mathrm{C} 17-\mathrm{N} 9$ & $107.4(3)$ \\
\hline $\mathrm{O} 10-\mathrm{Mo} 4-\mathrm{O} 11$ & $106.30(14)$ & $\mathrm{C} 18-\mathrm{C} 17-\mathrm{H} 17$ & 126.3 \\
\hline $\mathrm{O} 10-\mathrm{Mo} 4-\mathrm{O} 12$ & $99.66(12)$ & N9- $\mathrm{C} 17-\mathrm{H} 17$ & 126.3 \\
\hline $\mathrm{O} 11-\mathrm{Mo} 4-\mathrm{O} 12$ & $101.36(12)$ & $\mathrm{C} 17-\mathrm{C} 18-\mathrm{N} 10$ & $106.6(3)$ \\
\hline $\mathrm{O} 10-\mathrm{Mo} 4-\mathrm{O} 9$ & $100.30(12)$ & $\mathrm{C} 17-\mathrm{C} 18-\mathrm{H} 18$ & 126.7 \\
\hline $\mathrm{O} 11-\mathrm{Mo} 4-\mathrm{O} 9$ & $99.31(12)$ & $\mathrm{N} 10-\mathrm{C} 18-\mathrm{H} 18$ & 126.7 \\
\hline $\mathrm{O} 12-\mathrm{Mo} 4-\mathrm{O} 9$ & $145.80(9)$ & $\mathrm{N} 9-\mathrm{C} 19-\mathrm{N} 10$ & $108.1(3)$ \\
\hline $\mathrm{O} 10-\mathrm{Mo} 4-\mathrm{O} 24$ & $106.87(10)$ & $\mathrm{N} 9-\mathrm{C} 19-\mathrm{C} 20$ & $126.5(3)$ \\
\hline $\mathrm{O} 11-\mathrm{Mo} 4-\mathrm{O} 24$ & $146.81(11)$ & $\mathrm{N} 10-\mathrm{C} 19-\mathrm{C} 20$ & $125.3(3)$ \\
\hline $\mathrm{O} 12-\mathrm{Mo} 4-\mathrm{O} 24$ & $74.02(8)$ & $\mathrm{C} 19-\mathrm{C} 20-\mathrm{H} 20 \mathrm{~A}$ & 109.5 \\
\hline $\mathrm{O} 9-\mathrm{Mo} 4-\mathrm{O} 24$ & $73.74(8)$ & $\mathrm{C} 19-\mathrm{C} 20-\mathrm{H} 20 \mathrm{~B}$ & 109.5 \\
\hline $\mathrm{O} 10-\mathrm{Mo} 4-\mathrm{Mo5}$ & $93.77(10)$ & $\mathrm{H} 20 \mathrm{~A}-\mathrm{C} 20-\mathrm{H} 20 \mathrm{~B}$ & 109.5 \\
\hline $\mathrm{O} 11-\mathrm{Mo} 4-\mathrm{Mo5}$ & $135.81(10)$ & $\mathrm{C} 19-\mathrm{C} 20-\mathrm{H} 20 \mathrm{C}$ & 109.5 \\
\hline $\mathrm{O} 12-\mathrm{Mo} 4-\mathrm{Mo5}$ & $35.45(6)$ & $\mathrm{H} 20 \mathrm{~A}-\mathrm{C} 20-\mathrm{H} 20 \mathrm{C}$ & 109.5 \\
\hline $\mathrm{O} 9-\mathrm{Mo} 4-\mathrm{Mo5}$ & $115.58(6)$ & $\mathrm{H} 20 \mathrm{~B}-\mathrm{C} 20-\mathrm{H} 20 \mathrm{C}$ & 109.5 \\
\hline $\mathrm{O} 24-\mathrm{Mo} 4-\mathrm{Mo5}$ & $42.18(5)$ & $\mathrm{C} 19-\mathrm{N} 9-\mathrm{C} 17$ & $108.7(3)$ \\
\hline $\mathrm{O} 13-\mathrm{Mo5}-\mathrm{O} 14$ & $105.18(11)$ & $\mathrm{C} 19-\mathrm{N} 9-\mathrm{H} 9 \mathrm{~N}$ & $128.0(19)$ \\
\hline $\mathrm{O} 13-\mathrm{Mo5}-\mathrm{O} 15$ & $96.34(10)$ & $\mathrm{C} 17-\mathrm{N} 9-\mathrm{H} 9 \mathrm{~N}$ & $123.0(19)$ \\
\hline $\mathrm{O} 14-\mathrm{Mo5}-\mathrm{O} 15$ & $99.61(10)$ & $\mathrm{C} 19-\mathrm{N} 10-\mathrm{C} 18$ & $109.2(3)$ \\
\hline $\mathrm{O} 13-\mathrm{Mo5}-\mathrm{O} 12$ & $99.85(10)$ & $\mathrm{C} 19-\mathrm{N} 10-\mathrm{H} 10 \mathrm{~N}$ & $125.4(19)$ \\
\hline $\mathrm{O} 14-\mathrm{Mo5}-\mathrm{O} 12$ & $92.28(10)$ & $\mathrm{C} 18-\mathrm{N} 10-\mathrm{H} 10 \mathrm{~N}$ & $125.4(19)$ \\
\hline $\mathrm{O} 15-\mathrm{Mo5}-\mathrm{O} 12$ & $156.65(9)$ & $\mathrm{C} 22-\mathrm{C} 21-\mathrm{N} 11$ & $106.6(3)$ \\
\hline $\mathrm{O} 13-\mathrm{Mo5}-\mathrm{O} 24$ & $95.51(10)$ & $\mathrm{C} 22-\mathrm{C} 21-\mathrm{H} 21$ & 126.7 \\
\hline $\mathrm{O} 14-\mathrm{Mo5}-\mathrm{O} 24$ & $156.48(9)$ & $\mathrm{N} 11-\mathrm{C} 21-\mathrm{H} 21$ & 126.7 \\
\hline $\mathrm{O} 15-\mathrm{Mo5}-\mathrm{O} 24$ & $88.88(8)$ & $\mathrm{C} 21-\mathrm{C} 22-\mathrm{N} 12$ & $107.2(3)$ \\
\hline $\mathrm{O} 12-\mathrm{Mo5}-\mathrm{O} 24$ & $72.96(8)$ & $\mathrm{C} 21-\mathrm{C} 22-\mathrm{H} 22$ & 126.4 \\
\hline $\mathrm{O} 13-\mathrm{Mo5}-\mathrm{O} 22$ & $165.08(10)$ & $\mathrm{N} 12-\mathrm{C} 22-\mathrm{H} 22$ & 126.4 \\
\hline $\mathrm{O} 14-\mathrm{Mo5}-\mathrm{O} 22$ & $88.42(9)$ & $\mathrm{N} 12-\mathrm{C} 23-\mathrm{N} 11$ & $108.1(3)$ \\
\hline $\mathrm{O} 15-\mathrm{Mo5}-\mathrm{O} 22$ & $74.97(7)$ & $\mathrm{N} 12-\mathrm{C} 23-\mathrm{C} 24$ & $125.8(3)$ \\
\hline $\mathrm{O} 12-\mathrm{Mo5}-\mathrm{O} 22$ & $85.42(8)$ & $\mathrm{N} 11-\mathrm{C} 23-\mathrm{C} 24$ & $126.1(3)$ \\
\hline $\mathrm{O} 24-\mathrm{Mo5}-\mathrm{O} 22$ & $72.54(7)$ & $\mathrm{C} 23-\mathrm{C} 24-\mathrm{H} 24 \mathrm{~A}$ & 109.5 \\
\hline $\mathrm{O} 13-\mathrm{Mo5}-\mathrm{Mo} 4$ & $87.53(8)$ & $\mathrm{C} 23-\mathrm{C} 24-\mathrm{H} 24 \mathrm{~B}$ & 109.5 \\
\hline $\mathrm{O} 14-\mathrm{Mo5}-\mathrm{Mo} 4$ & $126.61(8)$ & $\mathrm{H} 24 \mathrm{~A}-\mathrm{C} 24-\mathrm{H} 24 \mathrm{~B}$ & 109.5 \\
\hline $\mathrm{O} 15-\mathrm{Mo5}-\mathrm{Mo} 4$ & $130.86(6)$ & $\mathrm{C} 23-\mathrm{C} 24-\mathrm{H} 24 \mathrm{C}$ & 109.5 \\
\hline $\mathrm{O} 12-\mathrm{Mo5}-\mathrm{Mo} 4$ & $34.41(6)$ & $\mathrm{H} 24 \mathrm{~A}-\mathrm{C} 24-\mathrm{H} 24 \mathrm{C}$ & 109.5 \\
\hline
\end{tabular}




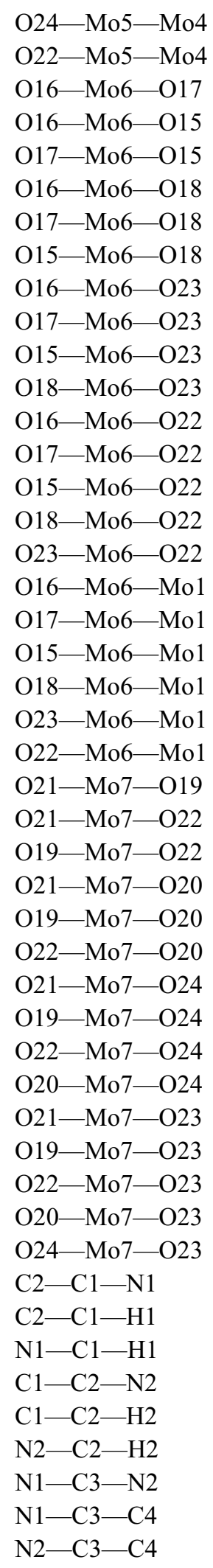

$42.08(5)$

$89.18(5)$

105.29 (12)

98.56 (10)

99.46 (10)

100.69 (10)

90.59 (9)

155.08 (8)

97.03 (10)

$154.60(9)$

$88.86(8)$

$73.23(8)$

166.13 (10)

87.49 (9)

$73.58(7)$

$84.24(8)$

$71.80(7)$

$90.80(8)$

$124.59(7)$

130.62 (6)

34.01 (6)

$41.78(5)$

$86.14(5)$

$103.66(11)$

$102.76(10)$

101.19 (9)

101.84 (10)

100.32 (9)

$142.21(8)$

$85.71(9)$

$170.59(8)$

$77.15(8)$

$76.58(8)$

$174.78(9)$

$81.55(8)$

$76.12(8)$

$76.77(8)$

89.07 (6)

106.8 (3)

126.6

126.6

106.9 (3)

126.6

126.6

108.0 (3)

$125.0(3)$

$127.0(3)$
$\mathrm{H} 24 \mathrm{~B}-\mathrm{C} 24-\mathrm{H} 24 \mathrm{C}$

C23-N11-C21

$\mathrm{C} 23-\mathrm{N} 11-\mathrm{H} 11 \mathrm{~N}$

C21-N11-H11N

$\mathrm{C} 23-\mathrm{N} 12-\mathrm{C} 22$

$\mathrm{C} 23-\mathrm{N} 12-\mathrm{H} 12 \mathrm{~N}$

$\mathrm{C} 22-\mathrm{N} 12-\mathrm{H} 12 \mathrm{~N}$

$\mathrm{C} 26-\mathrm{C} 25-\mathrm{N} 13$

$\mathrm{C} 26-\mathrm{C} 25-\mathrm{H} 25$

$\mathrm{N} 13-\mathrm{C} 25-\mathrm{H} 25$

$\mathrm{C} 25-\mathrm{C} 26-\mathrm{N} 14$

$\mathrm{C} 25-\mathrm{C} 26-\mathrm{H} 26$

$\mathrm{N} 14-\mathrm{C} 26-\mathrm{H} 26$

$\mathrm{N} 14-\mathrm{C} 27-\mathrm{N} 13$

$\mathrm{N} 14-\mathrm{C} 27-\mathrm{C} 28$

$\mathrm{N} 13-\mathrm{C} 27-\mathrm{C} 28$

$\mathrm{C} 27-\mathrm{C} 28-\mathrm{H} 28 \mathrm{~A}$

$\mathrm{C} 27-\mathrm{C} 28-\mathrm{H} 28 \mathrm{~B}$

$\mathrm{H} 28 \mathrm{~A}-\mathrm{C} 28-\mathrm{H} 28 \mathrm{~B}$

$\mathrm{C} 27-\mathrm{C} 28-\mathrm{H} 28 \mathrm{C}$

$\mathrm{H} 28 \mathrm{~A}-\mathrm{C} 28-\mathrm{H} 28 \mathrm{C}$

$\mathrm{H} 28 \mathrm{~B}-\mathrm{C} 28-\mathrm{H} 28 \mathrm{C}$

$\mathrm{C} 27-\mathrm{N} 13-\mathrm{C} 25$

$\mathrm{C} 27-\mathrm{N} 13-\mathrm{H} 13 \mathrm{~N}$

$\mathrm{C} 25-\mathrm{N} 13-\mathrm{H} 13 \mathrm{~N}$

$\mathrm{C} 27-\mathrm{N} 14-\mathrm{C} 26$

$\mathrm{C} 30-\mathrm{C} 29-\mathrm{N} 15$

$\mathrm{C} 30-\mathrm{C} 29-\mathrm{H} 29$

N15-C29- 29

$\mathrm{C} 29-\mathrm{C} 30-\mathrm{N} 16$

$\mathrm{C} 29-\mathrm{C} 30-\mathrm{H} 30$

$\mathrm{N} 16-\mathrm{C} 30-\mathrm{H} 30$

N16-C31-N15

$\mathrm{N} 16-\mathrm{C} 31-\mathrm{C} 32$

$\mathrm{N} 15-\mathrm{C} 31-\mathrm{C} 32$

$\mathrm{C} 31-\mathrm{C} 32-\mathrm{H} 32 \mathrm{~A}$

C $31-\mathrm{C} 32-\mathrm{H} 32 \mathrm{~B}$

$\mathrm{H} 32 \mathrm{~A}-\mathrm{C} 32-\mathrm{H} 32 \mathrm{~B}$

C31-C32- $332 \mathrm{C}$

$\mathrm{H} 32 \mathrm{~A}-\mathrm{C} 32-\mathrm{H} 32 \mathrm{C}$

$\mathrm{H} 32 \mathrm{~B}-\mathrm{C} 32-\mathrm{H} 32 \mathrm{C}$

C31-N15-C29

$\mathrm{C} 31-\mathrm{N} 15-\mathrm{H} 15 \mathrm{~N}$

$\mathrm{C} 29-\mathrm{N} 15-\mathrm{H} 15 \mathrm{~N}$

$\mathrm{C} 31-\mathrm{N} 16-\mathrm{C} 30$

$\mathrm{H} 25 \mathrm{~V}-\mathrm{O} 25-\mathrm{H} 25 \mathrm{~W}$

$\mathrm{H} 26 \mathrm{~V}-\mathrm{O} 26-\mathrm{H} 26 \mathrm{~W}$
109.5

109.3 (2)

125.3 (18)

125.4 (18)

108.8 (2)

124.9 (19)

125.9 (19)

105.5 (4)

127.2

127.2

109.8 (5)

125.1

125.1

109.4 (4)

126.1 (4)

124.5 (4)

109.5

109.5

109.5

109.5

109.5

109.5

108.9 (4)

$126(2)$

125 (2)

106.3 (4)

105.7 (3)

127.1

127.1

109.6 (3)

125.2

125.2

110.4 (3)

125.2 (3)

124.4 (3)

109.5

109.5

109.5

109.5

109.5

109.5

108.1 (3)

127.1 (19)

124.8 (19)

106.1 (3)

105 (3)

107 (4) 
Hydrogen-bond geometry $\left(\AA,{ }^{\circ}\right)$

\begin{tabular}{|c|c|c|c|c|}
\hline$D-\mathrm{H} \cdots A$ & $D-\mathrm{H}$ & $\mathrm{H} \cdots A$ & $D \cdots A$ & $D-\mathrm{H} \cdots A$ \\
\hline $\mathrm{N} 1-\mathrm{H} 1 N \cdots \mathrm{O} 22^{\mathrm{i}}$ & $0.88(2)$ & $1.85(2)$ & $2.710(3)$ & $167(2)$ \\
\hline $\mathrm{N} 2-\mathrm{H} 2 N \cdots \mathrm{O} 20$ & $0.87(2)$ & $1.79(2)$ & $2.655(3)$ & $172(2)$ \\
\hline $\mathrm{N} 3-\mathrm{H} 3 N \cdots \mathrm{O} 18^{\mathrm{i}}$ & $0.88(2)$ & $1.87(2)$ & $2.743(4)$ & $171(5)$ \\
\hline $\mathrm{N} 4-\mathrm{H} 4 N \cdots \mathrm{O} 9$ & $0.88(2)$ & $1.79(2)$ & $2.662(4)$ & $171(5)$ \\
\hline $\mathrm{N} 5-\mathrm{H} 5 N \cdots \mathrm{O} 12$ & $0.89(2)$ & $1.77(3)$ & $2.644(4)$ & $168(4)$ \\
\hline $\mathrm{N} 6-\mathrm{H} 6 N \cdots \mathrm{O} 3^{\mathrm{ii}}$ & $0.90(2)$ & $1.80(3)$ & $2.684(4)$ & $168(5)$ \\
\hline $\mathrm{N} 7-\mathrm{H} 7 N \cdots \mathrm{O} 1^{\mathrm{iii}}$ & $0.89(3)$ & $1.83(3)$ & $2.697(4)$ & $167(3)$ \\
\hline $\mathrm{N} 8-\mathrm{H} 8 N \cdots \mathrm{O} 25$ & $0.89(3)$ & $1.78(3)$ & $2.659(4)$ & $172(5)$ \\
\hline $\mathrm{N} 9-\mathrm{H} 9 N \cdots \mathrm{N} 14^{\mathrm{iv}}$ & $0.89(2)$ & $1.81(2)$ & $2.698(4)$ & $177(3)$ \\
\hline $\mathrm{N} 10-\mathrm{H} 10 N \cdots \mathrm{O} 15$ & $0.88(2)$ & $1.98(2)$ & $2.852(3)$ & $174(3)$ \\
\hline $\mathrm{N} 11-\mathrm{H} 11 N \cdots \mathrm{O} 6$ & $0.88(2)$ & $1.78(2)$ & $2.636(3)$ & $166(3)$ \\
\hline $\mathrm{N} 12-\mathrm{H} 12 N \cdots \mathrm{N} 16^{\mathrm{v}}$ & $0.88(2)$ & $1.87(2)$ & $2.725(4)$ & $165(3)$ \\
\hline $\mathrm{N} 13-\mathrm{H} 13 N \cdots \mathrm{O} 5$ & $0.88(4)$ & $2.32(4)$ & $3.186(5)$ & $167(3)$ \\
\hline $\mathrm{N} 13-\mathrm{H} 13 N \cdots \mathrm{O} 8$ & $0.88(4)$ & $2.55(4)$ & $3.043(5)$ & $116(2)$ \\
\hline $\mathrm{N} 15-\mathrm{H} 15 N \cdots \mathrm{O} 14$ & $0.87(3)$ & $2.22(3)$ & $2.953(4)$ & $142(3)$ \\
\hline $\mathrm{N} 15-\mathrm{H} 15 N \cdots \mathrm{O} 17$ & $0.87(3)$ & $2.27(3)$ & $2.917(4)$ & $132(3)$ \\
\hline $\mathrm{O} 25-\mathrm{H} 25 V \cdots \mathrm{O} 19$ & $0.86(2)$ & $2.00(2)$ & $2.788(3)$ & $154(5)$ \\
\hline $\mathrm{O} 25-\mathrm{H} 25 W \cdots \mathrm{O} 26^{\mathrm{vi}}$ & $0.86(3)$ & $1.85(3)$ & $2.702(4)$ & $176(5)$ \\
\hline $\mathrm{O} 26-\mathrm{H} 26 V \cdot \cdots \mathrm{O} 10$ & $0.83(4)$ & $2.50(6)$ & $2.999(4)$ & $119(5)$ \\
\hline $\mathrm{O} 26-\mathrm{H} 26 V \cdots \mathrm{O} 13$ & $0.83(4)$ & $2.25(5)$ & $3.009(4)$ & $151(6)$ \\
\hline $\mathrm{O} 26-\mathrm{H} 26 W \cdots \mathrm{O} 7$ & $0.83(4)$ & $1.99(6)$ & $2.737(4)$ & $149(6)$ \\
\hline $\mathrm{C} 4-\mathrm{H} 4 A \cdots \mathrm{O} 8$ & 0.98 & 2.35 & $3.228(5)$ & 149 \\
\hline $\mathrm{C} 8-\mathrm{H} 8 B \cdots \mathrm{O} 2^{\mathrm{i}}$ & 0.98 & 2.54 & $3.517(6)$ & 176 \\
\hline $\mathrm{C} 8-\mathrm{H} 8 \mathrm{C} \cdots \mathrm{O} 8$ & 0.98 & 2.49 & $3.386(5)$ & 152 \\
\hline $\mathrm{C} 10-\mathrm{H} 10 \cdots \mathrm{O} 26^{\text {vii }}$ & 0.95 & 2.45 & $3.318(5)$ & 151 \\
\hline $\mathrm{C} 12-\mathrm{H} 12 A \cdots \mathrm{O} 5^{\mathrm{ii}}$ & 0.98 & 2.44 & $3.407(5)$ & 167 \\
\hline $\mathrm{C} 13-\mathrm{H} 13 \cdots \mathrm{O} 4^{\mathrm{iii}}$ & 0.95 & 2.50 & $3.297(6)$ & 141 \\
\hline $\mathrm{C} 17-\mathrm{H} 17 \cdots \mathrm{O} 2^{\text {viii }}$ & 0.95 & 2.46 & $3.154(4)$ & 130 \\
\hline $\mathrm{C} 18-\mathrm{H} 18 \cdots \mathrm{O} 26$ & 0.95 & 2.58 & $3.522(5)$ & 172 \\
\hline $\mathrm{C} 21-\mathrm{H} 21 \cdots \mathrm{O} 16$ & 0.95 & 2.46 & $3.302(4)$ & 147 \\
\hline $\mathrm{C} 22-\mathrm{H} 22 \cdots \mathrm{O} 11^{\text {ix }}$ & 0.95 & 2.22 & $3.113(4)$ & 156 \\
\hline $\mathrm{C} 24-\mathrm{H} 24 B \cdots \mathrm{O} 7$ & 0.98 & 2.50 & $3.346(5)$ & 145 \\
\hline $\mathrm{C} 25-\mathrm{H} 25 \cdots \mathrm{O} 8$ & 0.95 & 2.59 & $3.055(5)$ & 110 \\
\hline $\mathrm{C} 32-\mathrm{H} 32 B \cdots \mathrm{O} 14$ & 0.98 & 2.55 & $3.234(5)$ & 126 \\
\hline
\end{tabular}

Symmetry codes: (i) $-x+1 / 2, y-1 / 2,-z+1 / 2$; (ii) $-x+1 / 2, y+1 / 2,-z+1 / 2$; (iii) $x+1 / 2,-y+1 / 2, z-1 / 2$; (iv) $-x+1 / 2, y+1 / 2,-z+3 / 2 ;$ (v) $-x+1 / 2, y-1 / 2,-z+3 / 2$; (vi) $x-1 / 2,-y+1 / 2, z-1 / 2$; (vii) $-x+1,-y+1,-z+1$; (viii) $x+1 / 2,-y+1 / 2, z+1 / 2$; (ix) $x-1 / 2,-y+1 / 2, z+1 / 2$. 